\title{
Recent Advances in Brazing Fillers for Joining of Dissimilar Materials
}

\author{
Byungmin Ahn ${ }^{1,2}$ (D)
}

check for

updates

Citation: Ahn, B. Recent Advances in Brazing Fillers for Joining of Dissimilar Materials. Metals 2021, 11, 1037. https://doi.org/10.3390/ met11071037

Academic Editor: Russell Goodall

Received: 25 May 2021

Accepted: 23 June 2021

Published: 29 June 2021

Publisher's Note: MDPI stays neutral with regard to jurisdictional claims in published maps and institutional affiliations.

Copyright: (C) 2021 by the author. Licensee MDPI, Basel, Switzerland. This article is an open access article distributed under the terms and conditions of the Creative Commons Attribution (CC BY) license (https:/ / creativecommons.org/licenses/by/ $4.0 /)$.
1 Department of Materials Science and Engineering, Ajou University, Suwon 16499, Gyeonggi, Korea; byungmin@ajou.ac.kr; Tel.: +82-31-219-3531; Fax: +82-31-219-1613

2 Department of Energy Systems Research, Ajou University, Suwon 16499, Gyeonggi, Korea

\begin{abstract}
Brazing fillers for joining applications are essential for manufacturing and designing advanced materials. Several types of brazing fillers have been developed in recent decades to join similar or different engineering materials. Important parts of automotive and aircraft components, including steel, are often joined by brazing. In addition, ceramic components in microwave devices and circuits have been joined with a high level of integration in microelectronic devices. Similarly, in the medical field, metallic implants have been brazed to ceramic dental crowns. These advances have made human life more convenient. However, in brazing, there are certain issues with intermetallic compound (IMC) formation and residual stresses in joints at high temperatures. Nanoparticlereinforced fillers have been proposed to control IMCs, but there are other dispersion and particle segregation issues at the joints. In this study, various types of brazing fillers, joint fabrication processes, and brazing technologies developed in recent decades are reviewed. Furthermore, new developments in brazing materials and their specific applications are presented. Finally, the emerging areas in brazing, including the recent entropy-modified brazing fillers for various structural and technological fields, are discussed.
\end{abstract}

Keywords: brazing; joining; dissimilar; laser; automotive; melting; joint

\section{Introduction}

Almost 5000 years old, brazing as a joining technology is still useful for joining advanced materials and devices. In this process, two materials are joined with a filler material and heated above the melting point of the filler, which then solidifies and joins the contacting materials. The importance of brazing lies in the fact that two different materials, such as metals and ceramics, can also be joined [1]. The final joint characteristics depend on the parent materials and filler wettability on the contact surfaces. The melting point of the brazing filler must be lower than the solidus of the contact materials. Brazing fillers have a higher liquidus temperature $\left(>450{ }^{\circ} \mathrm{C}\right)$ than their low-temperature solder alloy counterparts. Low-temperature solder alloys for joining electronic materials and interconnections have a liquidus temperature of $<450{ }^{\circ} \mathrm{C}$ [2].

The principal advantage of brazing in modern manufacturing is that it can form strong bonds with dissimilar and completely different parent materials with minimal modification of the contact materials. By contrast, high-temperature welding produces a stronger bond but requires two similar parent metals, and intensive local heating causes distortion and stresses, as well as the creation of heat-affected zones (HAZ) [2,3]. Mechanical fasteners using bolt-nut connections may face problems of loosening, decreased clamping force, and low fatigue strength due to the uneven load distribution and stress concentrations at the threads $[4,5]$. The increased use of fillers in automotive and aerospace applications, microwave devices, and machines exposed to harsh environments necessitates the development of brazing fillers. Several complex structures, ceramics, and metals are usually required for joining electronics, sensors, and transducers, microwave devices, and medical and energy storage applications [6-9]. 
This study focuses on the recent advances in brazing alloys for the joining of various similar and dissimilar material combinations with an emphasis on the brazing of metals and ceramics. We reviewed different filler materials for various applications, including the recently discovered high-entropy alloys (HEAs) in brazing. HEAs have shown desirable properties for brazing applications, such as good wetting and minimal reaction compounds, which have led to significant enhancements in the strength of brazed joints under high temperatures and harsh service conditions [10,11]. HEA-based fillers are unique because the resulting brazed joint is composed of solid solution phases, which accommodate the brazing seam and are beneficial for minimizing the coefficient of thermal expansion mismatch induced between the contact materials [12].

The rest of this paper is organized as follows: Section 2 discusses the various methods of brazing, wetting theory, thermodynamic considerations, and interfacial reactions in brazing technology. Section 3 describes the relevant criteria of brazing fillers in the literature and their recent applications in brazing. Section 4 presents the development trends of filler materials. Section 5 provides an overview of various trends in joining dissimilar materials. Section 6 highlights the emerging areas and applications of brazing. Section 7 focuses on HEAs in brazing, and, finally, Section 8 presents the summary and conclusions.

\section{Brazing Methods and Trend}

The steps involved in the brazing process include joint formation using different energy sources to melt the fillers and establish the bond. Table 1 summarizes the various brazing methods and their advantages and drawbacks, citing popular applications and requirements.

Table 1. Overview of various brazing methods, their advantages, drawbacks, and applications [1,2].

\begin{tabular}{ccccc}
\hline Process & Detail & Advantages & Drawbacks & Applications \\
\hline Flame brazing & $\begin{array}{c}\text { The joint fixed in jigs is } \\
\text { heated by a gas flame }\end{array}$ & $\begin{array}{c}\text { Cheap and fast; limited } \\
\text { damage }\end{array}$ & $\begin{array}{c}\text { Needs a highly skilled } \\
\text { operator }\end{array}$ & $\begin{array}{c}\text { Low volume; low heating } \\
\text { temperatures }\end{array}$ \\
\hline Induction brazing & $\begin{array}{c}\text { Electromagnetic } \\
\text { induction heating }\end{array}$ & $\begin{array}{c}\text { Highly efficient; localized } \\
\text { heating; multiple parts } \\
\text { can be completed }\end{array}$ & $\begin{array}{c}\text { Multicomponent } \\
\text { shapes are not possible }\end{array}$ & $\begin{array}{c}\text { Generally suitable for } \\
\text { highly resistive metals } \\
\text { and steels }\end{array}$ \\
\hline $\begin{array}{c}\text { Furnace/vacuum } \\
\text { brazing }\end{array}$ & $\begin{array}{c}\text { Self-supported joint } \\
\text { passed in the furnace } \\
\text { with or without a } \\
\text { controlled atmosphere }\end{array}$ & $\begin{array}{c}\text { Automated; mass } \\
\text { production; limited } \\
\text { voiding under a } \\
\text { controlled atmosphere }\end{array}$ & $\begin{array}{c}\text { High heating and } \\
\text { maintenance cost; batch } \\
\text { process; clean parts } \\
\text { required }\end{array}$ & $\begin{array}{c}\text { Different geometries; } \\
\text { suitable for stable oxide } \\
\text { metals such as Ti and Al }\end{array}$ \\
\hline Laser brazing & $\begin{array}{c}\text { The laser beam quickly } \\
\text { melts the filler, with no } \\
\text { need for flux }\end{array}$ & $\begin{array}{c}\text { Automated; mass } \\
\text { production; high joint } \\
\text { strength }\end{array}$ & $\begin{array}{c}\text { Tooling is rather } \\
\text { expensive but can be } \\
\text { compromised with } \\
\text { faster and robust joints }\end{array}$ & $\begin{array}{c}\text { Suitable for most metals } \\
\text { and ceramics }\end{array}$ \\
\hline
\end{tabular}

Among the above methods, flame brazing is the cheapest, but a highly skilled operator is required. Flame brazing employs an oxyacetylene gas torch to melt and braze the filler metal inside the joint and attain bonding. Induction brazing is more suitable for similar materials. Furnace or vacuum brazing is the most popular method for automated joining of similar or dissimilar materials under a controlled environment and is suitable for mass production [1,2]. Currently, brazing industries are moving toward laser brazing owing to its sound and robust joint and brazing operation [3].

Figure 1 shows the various steps involved in the joining and forming of a sound metallurgical interface between dissimilar materials. There should be an appropriate gap between the joint materials, as indicated in step 1. Proper cleaning of the contact surfaces is necessary before brazing. The oxidized layers on the two surfaces are removed by applying a brazing flux (step 3). The brazing filler is melted above its liquidus temperature, which wets the contact surfaces and often flows between the components to be joined (step 4). In the final step, the filler cools, solidifies, and bonds the components (step 5) $[2,13,14]$. 


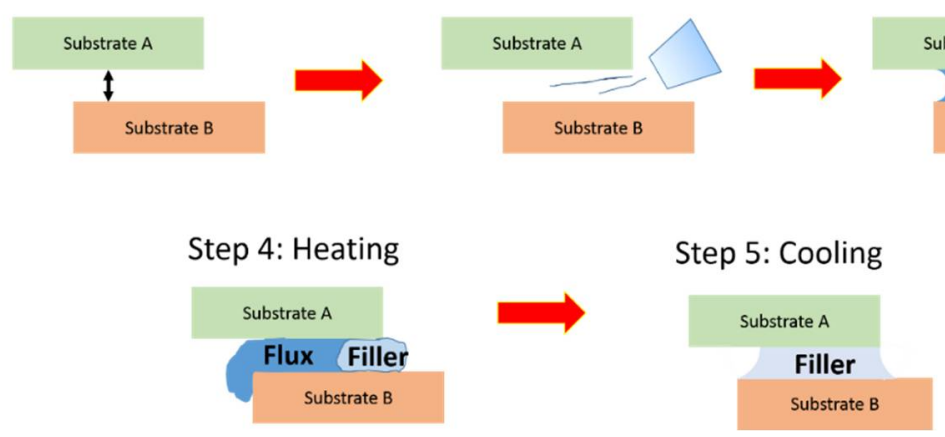

Figure 1. Various steps of the brazing process.

\subsection{Wetting Theory}

The wettability of brazing fillers is the most important property for brazing. It indicates the fluidity of the molten filler on the contact surfaces. The melt fluidity determines the penetration of the filler metal across and inside the joint surface. A free-flowing filler metal is necessary for most brazing applications, except where metal placement in the joint is difficult.

However, for a wide joint gap, the melt fluidity should not be extremely high; otherwise, it may fail to stay inside the joint and flow down the joint during brazing [15]. This may further cause the formation of cracks and pores in the final joint. Therefore, most brazing fillers have a single melting temperature or eutectic composition for optimal fluidity at the brazing temperature. In multicomponent alloys with multiple phases, it is difficult to have a single melting point, and some phases remain in a partially molten state, causing sluggish fluidity and poor wetting $[15,16]$. This is undesirable, except when the joint clearance is wide and filler metal retention is difficult.

\subsection{Thermodynamic Considerations}

Various types of forces are exerted by the surface tensions at the three interfaces, as shown in Figure 2. These force vectors are known as the liquid-surface interface tension $\left(\gamma_{S L}\right)$, liquid-vapor interface tension $\left(\gamma_{L V}\right)$, and solid-vapor interface tension $\left(\gamma_{S V}\right)$. Therefore, the net wetting force can be given by the following equation [17]:

$$
\mathrm{F}_{\text {net }}=\gamma_{S V}-\gamma_{S L} .
$$

In the equilibrium condition, the overall force is zero; therefore, $F_{\text {net }}=0$. The surface tension of the molten filler metal is in equilibrium with its horizontal component, that is,

$$
\gamma_{L V}=\gamma_{L V} \cos \theta=\mathrm{F}_{\text {net }}
$$

Equations (1) and (2),

$$
\gamma_{S V}=\gamma_{S L}+\gamma_{L V} \cos \theta
$$

After rearranging Equation (3), we obtain the popular Young equation:

$$
\gamma_{S L}=\gamma_{S V}-\gamma_{L V} \cos \theta .
$$

The angle subtended by the molten metal drop at the substrate is denoted as the wetting or contact angle. As illustrated in Figure 2, the wetting angle decreases with an increase in the contact area, which indicates better wettability [18]. In general, if $\theta<90^{\circ}$, the molten metal will wet the substrate sufficiently. By contrast, if $\theta>90^{\circ}$, the molten filler has poor wetting or non-wetting ability. It can be concluded that adhesive forces are associated with a lower wetting angle [19]. 


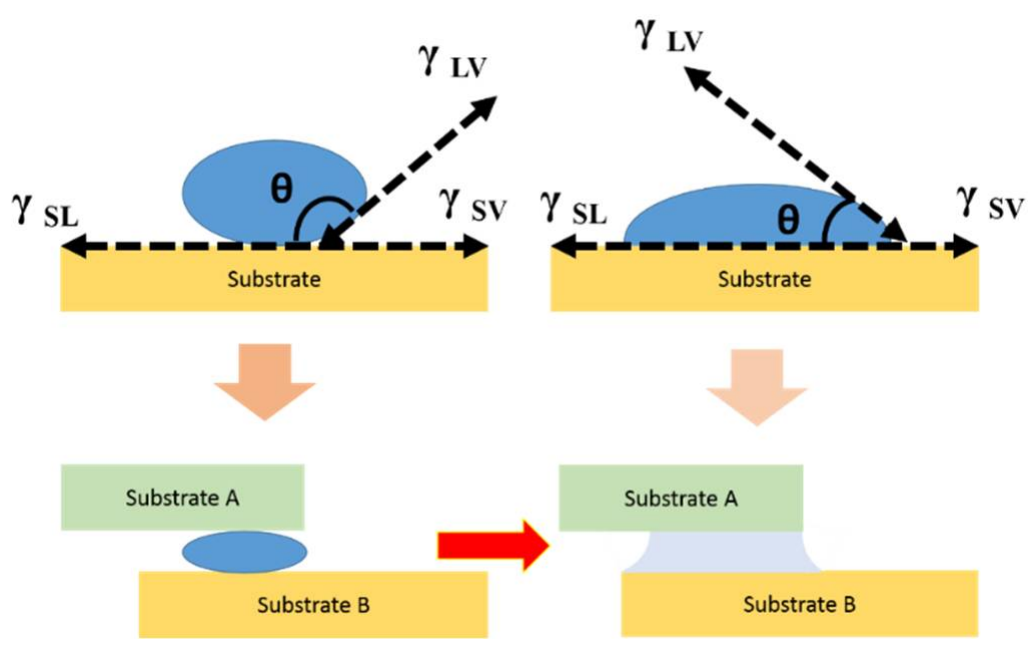

Figure 2. Schematic of wettability of filler on contact substrates. When $\theta>90^{\circ}$, wetting is insufficient; when $\theta<90^{\circ}$, wetting is sufficient. The cross-section of lap joints between two substrates is also shown at the bottom.

\subsection{Interfacial Reactions}

The joint formation during brazing requires a wettable filler metal to create a successful joint via the formation of different interface types during the brazing process. The reactions occurring at the joint interface can be classified into two major categories (Figure 3a,b).
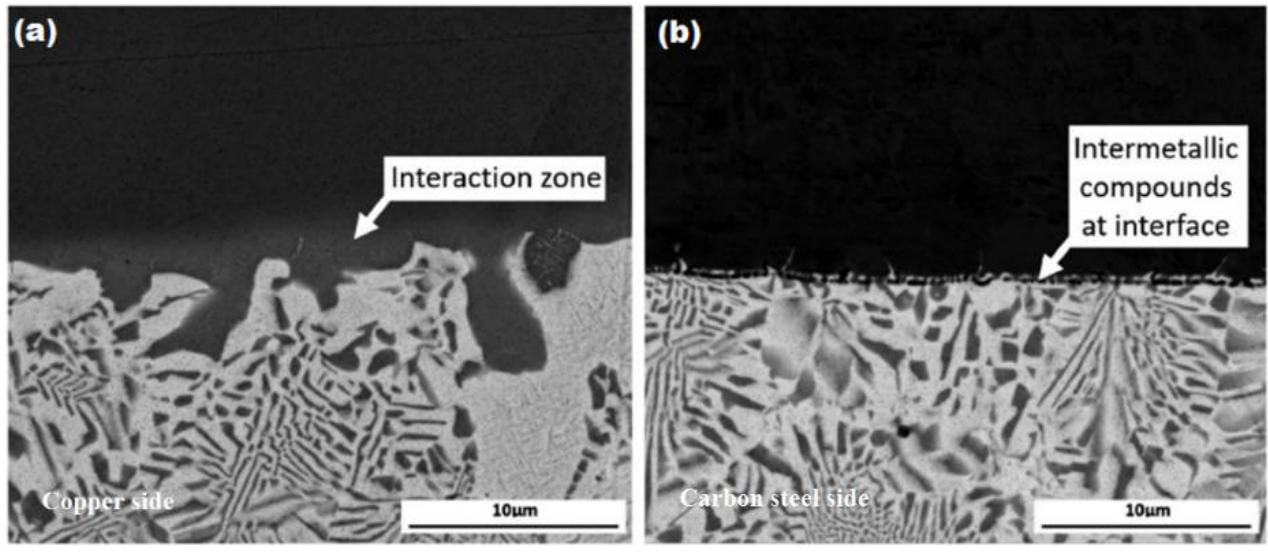

Figure 3. SEM image showing the interface between Ag-155 filler metal and (a) copper and (b) carbon steel [14].

An interfacial region forms as a result of the solubility of the filler elements within the joint or dissolution of the base substrate in the filler metal. Dissolution of the base materials may cause erosion wear if it occurs vigorously during brazing, leading to weaker joints. As depicted in Figure 3a, the filler penetrates the $\mathrm{Cu}$ base metal, while fine intermetallic compounds (IMCs) are produced when $\mathrm{Cu}$ is replaced with carbon steel (Figure 3b) [14]. The reaction of the filler metal with the base materials is more prominent in active metal brazing. For example, in ceramic to metal brazing, Shin et al. showed that the addition of Ti and $\mathrm{Sn}$ in a $\mathrm{Ag}$-Cu filler promotes the wettability of the $\mathrm{ZrO}_{2}$ ceramic because of the formation of IMCs between the brazing filler and contact surfaces [20]. However, Jung et al. reported that the overall joint strength depends on the thickness of the IMCs across the joint formed with an Al-based filler [21,22].

The most popular process used in aerospace is the brazing of Ti alloys, particularly Ti6Al4V and Al alloy components such as 5A06 Al alloy [23], A6061-T6 Al alloy [24], and $5052 \mathrm{Al}$ alloy [25]. Joining of a Ti skin to $\mathrm{Al}$ honeycomb structures in aviation industries 
is necessary to produce a balanced set of properties (lightweight $\mathrm{Al}$ and high stiffness Ti skin) [26]. Therefore, knowledge of interfaces is of paramount importance in airplanes for safety reasons [27]. Takemoto et al. [28] investigated the joining of Al to Ti using an Al-based brazing alloy. They reported that the inclusions of Si have a significant impact on interface growth. The interfacial region was reduced until $10 \mathrm{at} \% \mathrm{Si}$ was added, beyond which $\mathrm{Ti}_{7} \mathrm{Al}_{5} \mathrm{Si}_{12}$ emerged at the interface. Similar results were observed by Chen et al., who used an Al-12Si alloy for brazing Al to Ti. In their study, Si diffused at the interface, but the formation of IMCs was affected by the applied heat input rather than the composition of the Al-12Si alloy [23].

As reported in several studies, the formation of interfaces and IMCs is of significant interest in brazing because these compounds and their geometries are crucial for the process. Other reports on interface studies involve ceramic systems in active metal brazing, such as $\mathrm{SiC}$ [29-31], diamond [32-34], $\mathrm{WC}[35,36], \mathrm{Al}_{2} \mathrm{O}_{3}$ [37,38], and $\mathrm{ZrO}_{2}$ [6,39]. It can be inferred from these investigations that studies related to the brazing joint interface in dissimilar systems include improvements in wetting and minimization of interfacial compounds by suitable filler metals on a case-by-case basis.

\section{Desirable Properties of Brazing Fillers}

The selection of brazing fillers is based on various factors such as the type of base material, service temperature, gap clearance, brazing method, filler shape and size, and regulations on the use of toxic materials in brazing. For strong bonding and metallurgical compatibility, similar properties of both parent contact materials and fillers are highly desirable [1,2]. The ability to wet and spread over contact surfaces for robust joining is the primary requirement for any brazing filler metal. Several active metal fillers containing $\mathrm{Ti}$, $\mathrm{Zr}, \mathrm{Sn}, \mathrm{Nb}$, Hf, etc., were developed in the past to improve the wettability of substrates. The minor alloying elements ( $\mathrm{Sn}$ and $\mathrm{Ti}$ ) in Al-Si alloys promote the wetting and fluidity of the brazing fillers $[40,41]$. However, these alloying elements work only when added at suitable amounts. The addition of a higher amount of active elements may cause pitting and degrade the joint characteristics [40]. Similarly, $\mathrm{Mg}$ has also been observed to promote the wettability of base metals in $\mathrm{Al}$ alloys. The presence of $\mathrm{Mg}$ in brazing fillers also assists in strengthening the mechanisms and improving the strain-hardening rate of various joining operations. Mg-containing Al alloys can be a replacement for lightweight and highstrength alloys and steels owing to their high ductility, toughness, and strain-hardening characteristics [42-44]. Such wetting promoters and modifiers can be successfully exploited to enhance the wettability of a ceramic surface during active metal brazing [45].

\subsection{Service Conditions}

The service conditions of the finished part and the surrounding environment also affect the joint characteristics. For example, multi-component brazing fillers may form a galvanic couple in harsh chemical environments and deform quickly. Vacuum brazing is a relatively inexpensive and excellent technique to avoid oxidation and impurity inclusions in joined parts. The level of vacuum can reach $\sim 10^{-3}$ to $10^{-5} \mathrm{mbar}$ [46]. Other common inert gases used in brazing are $\mathrm{N}_{2}, \mathrm{H}_{2}$, and Ar. It has been observed that the amount of Nocoloc flux can be sufficiently reduced when brazing is performed in a $\mathrm{N}_{2}$ atmosphere $[47,48]$. In addition, the use of reducing atmospheres causes the reduction of metal oxides to metal and hence prevents the build-up of an oxidizing layer on the parts [49].

\subsection{Brazing Method}

The brazing method used also influences the joint quality and processing cost. For example, excessive use of filler metals can be avoided in flame brazing compared to induction or furnace brazing. Often, the cost and production volume of joints must be matched to the appropriate filler metal and compatible brazing process. It has been reported that a brazing filler containing $\mathrm{Zn}$ is not suitable for vacuum brazing because of its highly volatile nature. Various post-processing treatments after brazing may also save time and 
energy. For instance, high-purity Ti can be successfully joined to $\mathrm{Al}_{2} \mathrm{O}_{3}$ by employing a hydride atmosphere $\left(\mathrm{TiH}_{2}\right)$; hence, the need for metallization, plating, and annealing can be eliminated [46]. In metal-ceramic brazing, the presence of interlayers affects the strength of the brazed joint. During active metal brazing, various interfacial layers containing CuTi and $\mathrm{Cu}_{2} \mathrm{Ti}$ are formed across Ti-6Al-4V/ $\mathrm{ZrO}_{2}$ [50-52]. In general, $\mathrm{Cu}_{2} \mathrm{Ti}$ is produced at a relatively low brazing temperature; its formation increases with an increase in temperature, resulting in the highest hardness [53]. The thickness of the IMC layers directly affects the brazed joint strength and follows a power law dependence. Therefore, controlling these reaction layers is important for achieving the best set of joint properties [54].

\subsection{Brazeability}

The brazing temperature also affects the extent of formation of reaction compounds and joint strength. A single melting temperature of brazing alloys is desirable for a freeflow melt. However, it also depends on the joint clearance and melting point of the base materials. The brazing temperature is also limited by the formation of undesirable microstructures, such as HAZ in base materials, which leads to a loss of functionality. Active metal brazing of $\mathrm{Cu}$ and $\mathrm{Al}_{2} \mathrm{O}_{3}$ with a $\mathrm{Ag}-\mathrm{Cu}-\mathrm{Zr}-\mathrm{Sn}$ filler indicates that superior bonding strength is achieved at a low brazing temperature [55]. In the case of $\mathrm{Si}_{3} \mathrm{~N}_{4} / \mathrm{Si}_{3} \mathrm{~N}_{4}$ joints made with a Ag-Cu-Ti-Mo filler, the IMC thickness increases with temperature [56]. According to Yang et al., brazing of $\mathrm{Al}_{2} \mathrm{O}_{3} / \mathrm{Ti}-6 \mathrm{Al}-4 \mathrm{~V}$ with a Ag-Cu-Ti-B filler shows the presence of different reaction products (i.e., $\mathrm{TiCu}, \mathrm{Ti}_{2} \mathrm{Cu}, \mathrm{Ti}(\mathrm{Cu}, \mathrm{Al})$, and $\mathrm{Ti}_{2}(\mathrm{Cu}, \mathrm{Al})$ ). The solidification speeds of all these reaction compounds depend on the temperature, time, and presence of alloying elements that affect the overall brazed joint strength [57]. Jiang et al. brazed AISI 304 stainless steel to fins using a Ni-based filler and found that the brazed strength increased with the brazing temperature. They correlated this change with the microstructural transformation of the brazed seam, where brittle phases were found to decline with the brazing temperature [58]. Recently, to control the IMC layer thickness, the authors also introduced ceramic nanoparticles $\left(\mathrm{ZrO}_{2}, \mathrm{SiC}\right)$ in low-temperature $\mathrm{Al}$ brazing and improved the joint strength properties [21,22].

\subsection{Joint Gap}

In addition to the filler wettability and type of base material, a sufficient joint gap between the contact materials is required for the filler metal to freely flow and wet the surfaces. A wide joint clearance will require a sluggish flowability of the filler metal compared to the free flowability in a narrow joint clearance. The joint strength improves when the lap width is decreased. Elrefaey et al. performed vacuum brazing of a high-purity Ti plate and a low-carbon steel plate in a varying lap joint configuration using a Ag-based filler [53]. The authors suggested that a lap width of up to three times the base metal thickness is required for high-quality brazed joints [53,59]. There are various regulations on the use of certain elements in brazing fillers, such as Cd-containing brazing fillers, which are restricted for use in dairy equipment, food, and pharmaceuticals $[60,61]$. In certain cases, such as in the food, dairy, and biomedical sectors, $\mathrm{Cu}^{2+}$ concentrations greater than $0.5 \mathrm{mM}$ are known to cause toxicity to mesenchymal stem cells [62,63]. Similarly, Au nanoparticles have also shown some adverse effects on living cells beyond a certain level [64].

\section{Current Status in Various Brazing Filler Materials}

Filler metals are important in the brazing industry and differ in composition from the contact parent materials. Several specialized and miscellaneous alloys are being utilized commercially. Our study focused primarily on high-temperature solders or brazing fillers in general; however, understanding low-temperature solder fillers is useful. Therefore, they will be discussed briefly as well. 


\subsection{Low-Temperature Filler Metals}

The existing low-temperature fillers are mostly Sn-based alloys, such as binary $\mathrm{Sn}-\mathrm{Ag}$, $\mathrm{Sn}-\mathrm{Cu}$, and $\mathrm{Sn}-\mathrm{Bi}$, or ternary $\mathrm{Sn}-\mathrm{Bi}-\mathrm{Zn}$ and $\mathrm{Sn}-\mathrm{Ag}-\mathrm{Cu}[65]$. There is a range of melting temperatures of $\mathrm{Sn}$-based solders that exist for low-temperature soldering of microelectronic packaging devices. Conventional $\mathrm{Sn}-\mathrm{Pb}$ alloys are now avoided owing to their toxicity and the regulation of $\mathrm{Pb}$ in electronic devices [66]. The most popular alloy system substituted for the $\mathrm{Sn}-\mathrm{Pb}$ solder is $\mathrm{Sn}-\mathrm{Ag}-\mathrm{Cu}$, which is known as the SAC alloy, with a eutectic melting point of $217^{\circ} \mathrm{C}$. However, there are various challenges in the application of SAC solders to electronics, such as the formation of unwanted IMCs $\left(\mathrm{Cu}_{6} \mathrm{Sn}_{5}, \mathrm{Cu}_{3} \mathrm{Sn}\right.$, and $\mathrm{Ag}_{3} \mathrm{Sn}$ ), nucleation of $\mathrm{Sn}$ whiskers from the solder finish, and evolution of Kirkendall voids after long storage [67]. Various strategies to improve the solder joint reliability, such as the addition of alloying elements $(\mathrm{Ni}, \mathrm{Ag}, \mathrm{Cu}$, and $\mathrm{Ti}$ ) or nanoparticles inside the solder matrix to control the IMC growth, have been consistently explored [66]. However, the relatively poor mechanical strength and high melting point are still a concern among flexible interconnections. All these issues have triggered the development of solders with complex compositions. Recently, quaternary medium-entropy solders Sn-Bi-In-Zn [68] and Bi-Sn-In-Ga and quinary alloys have been developed for microelectronics [69]. It can be concluded that entropy-driven solders can be good substitutes for SAC alloys because they can minimize the formation of IMCs with the base metals.

\subsection{High-Temperature Brazing Fillers}

As already discussed, brazing fillers are generally high-temperature solders, where the melting point is greater than $450^{\circ} \mathrm{C}$. During brazing, the filler metal melts and produces a joint between the contact surfaces. Several hundreds of brazing fillers have been studied over recent decades. The most popular brazing alloys according to their class in ISO 17672:2016 are presented in Figure 4 [70]. This classification involves seven subfamilies and is designated according to the major elements constituting the family (Figure 4).

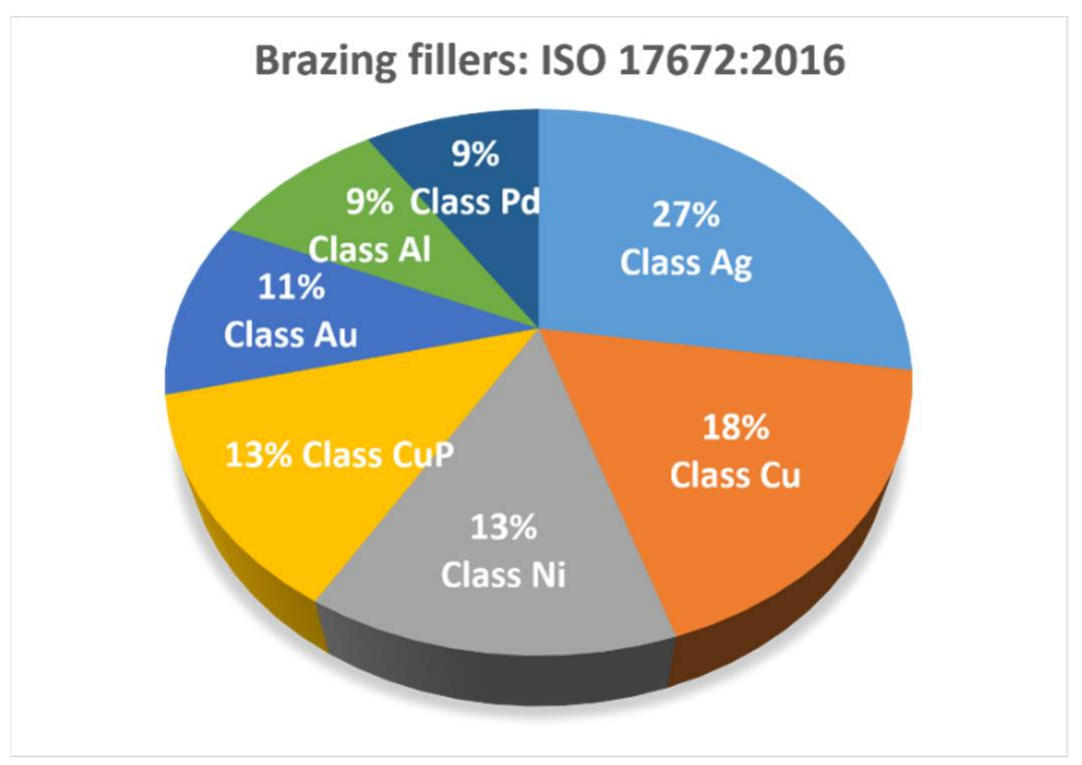

Figure 4. Various classes of brazing fillers according to ISO 17672:2016.

The seven subfamilies in each brazing filler class ( $\mathrm{Al}, \mathrm{Ag}, \mathrm{CuP}, \mathrm{Cu}, \mathrm{Ni}, \mathrm{Pd}$, and $\mathrm{Au}$ ) are summarized in Table 2. The particular applications of these alloys are also indicated. The copper-based fillers used for joining several dissimilar materials are divided into three variants.

Active metal brazing is currently becoming more popular owing to its ability to wet ceramic surfaces and combine two different materials across various disciplines [18]. Brazed joints have several advantages over mechanically fastened joints, including a reduction 
in the weight and size of the device, compactness, multiple-component brazing, and the use of high temperatures for thermal applications. In the past, many nanocomposite brazing alloys were also designed by some research groups where nanoparticles have shown enough potential to control the interface thickness and geometry and minimize thermal stresses [21,22]. However, the segregation of nanoparticles inside the joints is a critical issue that needs to be addressed via different material designs and procedures [71]. It is noted that each brazing alloy is different and contributes to the strength of brazed joints in particular applications. The effects of various brazing fillers on the mechanical properties of the joint strength are summarized in Table 3.

Table 2. Various classes of brazing alloys $[14,70]$.

\begin{tabular}{|c|c|c|}
\hline \multicolumn{3}{|c|}{ Standard Brazing Alloys According to ISO 17672:2016 } \\
\hline Filler Class & Components & Applications \\
\hline \multirow{2}{*}{ Class Al } & \multirow{2}{*}{ Al- and Mg-based alloys } & Joining of $\mathrm{Al}, \mathrm{Mg}$, and $\mathrm{Ti}$ components \\
\hline & & High $\mathrm{Mg}(001)$ for high-Mg alloys \\
\hline \multirow{3}{*}{ Class Ag } & \multirow{3}{*}{ Ag alloys } & Steel and stainless steels \\
\hline & & Copper, brass, and bronze alloys \\
\hline & & WC and diamond brazing \\
\hline Class CuP & $\mathrm{Cu}$ - and P-based filler metals & $\mathrm{Cu}$ alloys and fluxless brazing \\
\hline \multirow{3}{*}{ Class $\mathrm{Cu}$} & High-Cu alloys & Reducing gas furnace brazing (steel/WC) \\
\hline & $\mathrm{Cu}-\mathrm{Zn}$ alloys & $\mathrm{Cu}$, mild steel, and steel/WC \\
\hline & Inert gas brazing alloys & Galvanized steel joints for automotive \\
\hline \multirow{3}{*}{ Class Ni } & \multirow{3}{*}{$\mathrm{Ni}$ and Co alloys } & Stainless steels \\
\hline & & Ni- and Co-based superalloys \\
\hline & & Requires vacuum for corrosion-resistant joints \\
\hline \multirow{2}{*}{ Class Pd } & \multirow{2}{*}{ Pd alloys } & Aerospace and electronics \\
\hline & & Glass and chemical industries \\
\hline \multirow{2}{*}{ Class Au } & \multirow{2}{*}{ Au alloys } & Aerospace and electronics \\
\hline & & Jewelry and vacuum tubes \\
\hline \multicolumn{3}{|c|}{ Miscellaneous brazing fillers not included in ISO 17672:2016 } \\
\hline \multirow{2}{*}{ Special $\mathrm{Cu}$ alloys } & High-purity $\mathrm{Cu}$ alloys & $\begin{array}{l}\text { Ag filler alternative to carbon steel and stainless } \\
\text { steel brazing in reducing atmospheres }\end{array}$ \\
\hline & $\mathrm{Cu}-\mathrm{Mn}-\mathrm{Ni}$ & High-strength C steel/WC joint \\
\hline \multirow{2}{*}{ Pt alloys } & \multirow{2}{*}{ Various Pt-containing fillers } & Platinum jewelry \\
\hline & & Mo and $\mathrm{W}$ for ultrahigh-temperature applications \\
\hline Active metal fillers & $\begin{array}{l}\text { Ag alloys with active elements such as } \\
\text { Ti, Hf, and } \mathrm{Zr}\end{array}$ & Metal ceramic brazing \\
\hline Ti alloys & Ti with $\mathrm{Zr}, \mathrm{Cu}$, and Ni elements & Joining Ti in submarine and medical applications \\
\hline \multirow{4}{*}{ Refractory alloys } & $\mathrm{W}(80 \mathrm{Mo}-20 \mathrm{Ru} ; 65 \mathrm{Pd}-35 \mathrm{Co})$ & High-temperature systems \\
\hline & Mo (Hf-7Mo; Hf-40Mo) & Heating elements \\
\hline & $\mathrm{Nb}$ & Spacecraft propulsion systems \\
\hline & $\mathrm{Ta}(\mathrm{Ta}-\mathrm{V}-\mathrm{Nb} ; \mathrm{Ta}-\mathrm{V}-\mathrm{Ti})$ & Capacitors \\
\hline $\begin{array}{l}\text { Nanoparticle-reinforced brazing } \\
\text { alloys }\end{array}$ & $\begin{array}{l}\mathrm{Al}-\mathrm{Si}, \mathrm{Al}-\mathrm{Si}-\mathrm{Cu} \text {, and } \mathrm{Ag}-\mathrm{Cu}-\mathrm{Ti} \\
\text { reinforced with } \mathrm{ZrO}_{2}, \mathrm{CeO}_{2}, \mathrm{Al}_{2} \mathrm{O}_{3} \text {, } \\
\text { and } \mathrm{SiC} \text { nanoparticles }\end{array}$ & $\begin{array}{l}\text { Low-temperature Al brazing; ceramic-to-metal } \\
\text { brazing }\end{array}$ \\
\hline
\end{tabular}


Table 3. Current trends in important brazing materials and their effect on the mechanical properties of brazed joints.

\begin{tabular}{|c|c|c|c|c|c|c|}
\hline \multicolumn{2}{|c|}{ Contact Materials } & \multirow{2}{*}{$\begin{array}{c}\text { Brazing Filler } \\
\text { Sn10Ag4Ti }(w t \%)\end{array}$} & \multirow{2}{*}{$\begin{array}{l}\text { Brazing Condition } \\
900^{\circ} \mathrm{C} \text { for } 10 \mathrm{~min}\end{array}$} & \multirow{2}{*}{$\begin{array}{c}\text { Strength (MPa) } \\
\text { Four-point bending test, strength: } 57 \\
\mathrm{MPa}\end{array}$} & \multirow{2}{*}{$\begin{array}{c}\text { Application } \\
\text { Oil oxygen } \\
\text { burner in } \\
\text { petroleum } \\
\text { industry }\end{array}$} & \multirow{2}{*}{$\begin{array}{l}\text { Ref. } \\
{[72]}\end{array}$} \\
\hline $\begin{array}{l}\mathrm{Al}_{2} \mathrm{O}_{3} \\
(2003)\end{array}$ & $\begin{array}{l}\text { UMCo-50 } \\
\text { superalloys }\end{array}$ & & & & & \\
\hline $\begin{array}{l}\mathrm{Al}_{2} \mathrm{O}_{3} \\
(2003)\end{array}$ & Inconel 600, & Sn10Ag4Ti (wt\%) & $900^{\circ} \mathrm{C}$ for $10 \mathrm{~min}$ & $\begin{array}{l}\text { Four-point bending test, strength: } 69 \\
\qquad \mathrm{MPa}\end{array}$ & $\begin{array}{l}\text { Oil oxygen } \\
\text { burner in } \\
\text { petroleum } \\
\text { industry }\end{array}$ & {$[72]$} \\
\hline $\begin{array}{l}\mathrm{Al}_{2} \mathrm{O}_{3} \\
(2010)\end{array}$ & $\mathrm{Cu}$ & $\begin{array}{c}\mathrm{Cu}-72 \mathrm{wt} \% \mathrm{Ag}+(0.8-4.8 \\
\text { wt } \%) \mathrm{Ti}\end{array}$ & $\begin{array}{c}\text { Vacuum brazing, } 750{ }^{\circ} \mathrm{C}, 40 \mathrm{~min}, 840 \\
{ }^{\circ} \mathrm{C}, 15 \mathrm{~min}\end{array}$ & $\begin{array}{c}\text { Tensile strength, } 0.003 \mathrm{MPa} \text { at } 3 \\
\text { wt } \% \mathrm{Ti}, 840^{\circ} \mathrm{C}, 15 \mathrm{~min}\end{array}$ & $\begin{array}{l}\text { Metal-ceramic } \\
\text { joints }\end{array}$ & [73] \\
\hline $\begin{array}{l}\text { C fiber- } \\
\text { reinforced } \\
\text { SiC } \\
(2011)\end{array}$ & $\mathrm{Nb}$ & $\mathrm{Ti}_{39.4} \mathrm{Ni}_{39.4} \mathrm{Nb}_{21.2}(\mathrm{at} \%)$ & $\begin{array}{l}\text { Vacuum brazing, } 1160-1260^{\circ} \mathrm{C} \text { for } \\
10 \mathrm{~min}\end{array}$ & Shear strength: $149 \mathrm{MPa}, 1220^{\circ} \mathrm{C}$ & $\begin{array}{l}\text { Thrust } \\
\text { chamber in } \\
\text { rocket engines }\end{array}$ & [74] \\
\hline $\begin{array}{l}\mathrm{Al}_{2} \mathrm{O}_{3} \\
(2012)\end{array}$ & $\mathrm{Al}_{2} \mathrm{O}_{3}$ & $\begin{array}{l}\mathrm{Ag}-26.4 \mathrm{Cu}-4.5 \mathrm{Ti}+ \\
(0.35-1.5) \mathrm{B}+(0-5) \mathrm{TiH}_{2} \\
\text { (all in wt } \%)\end{array}$ & $900^{\circ} \mathrm{C}, 10 \mathrm{~min}$ & $\mathrm{~N} / \mathrm{A}$ & $\begin{array}{l}\text { High } \\
\text { temperature } \\
\text { applications }\end{array}$ & {$[75]$} \\
\hline $\begin{array}{l}\mathrm{Al} 3003 \\
(2012)\end{array}$ & $\mathrm{Al} 3003$ & $\mathrm{Zn}-(2-22) \mathrm{Al}($ wt. $\%)$ & Flame brazing, $500^{\circ} \mathrm{C}$ & $\begin{array}{l}\text { Joint hardness } 70-90 \mathrm{HV} \text {, tensile } \\
\text { strength: } 160 \mathrm{MPa}(2-82 \mathrm{wt} \% \mathrm{Al})\end{array}$ & $\begin{array}{l}\text { Automotive } \\
\text { parts }\end{array}$ & [76] \\
\hline $\begin{array}{l}\mathrm{Al}_{2} \mathrm{O}_{3} \\
(2013)\end{array}$ & Inconel 600 & $\begin{array}{c}92.75 \mathrm{Ag}-5.0 \mathrm{Cu}-1.0 \mathrm{Al}- \\
1.25 \mathrm{Ti} \\
(\mathrm{wt} . \%)\end{array}$ & Vacuum brazing, $950^{\circ} \mathrm{C}, 2 \mathrm{~min}$ & $\begin{array}{l}\text { Tensile shear strength: } 80 \mathrm{MPa} \text {, } \\
\text { fracture occurred on alumina during } \\
\text { tensile tensing }\end{array}$ & $\begin{array}{c}\text { Electrical } \\
\text { feedthrough in } \\
\text { nuclear plants }\end{array}$ & [77] \\
\hline $\begin{array}{l}\mathrm{Si}_{3} \mathrm{~N}_{4} \\
(2014)\end{array}$ & 42CrMo steel & $\begin{array}{c}\text { Ag54.67-Cu35.96-Ti4.37- } \\
\text { Pd5 } \\
\text { (at. \%) }\end{array}$ & $870-1020^{\circ} \mathrm{C}, 10-60 \mathrm{~min}$ & $\begin{array}{l}\text { Three-point bending test. Strength: } \\
352.3 \mathrm{MPa} \text { at } 920{ }^{\circ} \mathrm{C}, 30 \mathrm{~min}\end{array}$ & $\begin{array}{l}\text { Solid oxide fuel } \\
\text { cell sealing, } \\
\text { nuclear } \\
\text { reactors }\end{array}$ & [78] \\
\hline $\begin{array}{l}\text { C fiber- } \\
\text { reinforced } \\
\text { SiC } \\
(2014)\end{array}$ & AISI 304 & 48Ti-48Zr-4Be (wt.\%) & $\begin{array}{l}\text { Vacuum brazing, } 920-1050{ }^{\circ} \mathrm{C} \text { for } \\
15-90 \mathrm{~min}\end{array}$ & $\begin{array}{l}\text { Shear strength: } 109.13 \pm 2.55 \mathrm{MPa}, \\
\text { at } 950{ }^{\circ} \mathrm{C} / 60 \mathrm{~min}\end{array}$ & $\begin{array}{l}\text { High- } \\
\text { temperature } \\
\text { heat engines, } \\
\text { aerospace }\end{array}$ & [79] \\
\hline $\begin{array}{l}\text { Graphite } \\
(2014)\end{array}$ & $\mathrm{Cu}$ & $\mathrm{Ni}_{68.4} \mathrm{Cr}_{12.6} \mathrm{P}_{9} \mathrm{Cu}_{10}(\mathrm{wt} \%)$ & $900-1000^{\circ} \mathrm{C}, 10 \mathrm{~min}$ & Shear strength $60 \mathrm{MPa}, 950^{\circ} \mathrm{C}$ & $\begin{array}{l}\text { Plasma facing } \\
\text { materials }\end{array}$ & {$[80]$} \\
\hline $\begin{array}{c}5 \mathrm{~mol} \% \mathrm{Y}- \\
\mathrm{ZrO}_{2} \\
(2015)\end{array}$ & $\begin{array}{l}\text { AISI } 420 \\
\text { steel }\end{array}$ & $\begin{array}{c}\mathrm{Ag} / \mathrm{Ni} \text { nanopaste }(1: 0.8 \\
\mathrm{wt} \% \text {, particle size } 80 \mathrm{~nm})\end{array}$ & $270-360^{\circ} \mathrm{C}, 60-150 \mathrm{~min}$ & $\begin{array}{l}\text { Shear strength: } 23 \pm 1 \mathrm{MPa} \text { at } 270 \\
{ }^{\circ} \mathrm{C}, 120 \mathrm{~min}\end{array}$ & Turbine blades & [81] \\
\hline $\begin{array}{l}\mathrm{Si}_{3} \mathrm{~N}_{4} \\
(2016)\end{array}$ & $\begin{array}{c}\text { Invar } \\
(\mathrm{Fe}-32 \mathrm{Ni}) \\
\text { wt } \%\end{array}$ & $\begin{array}{l}\text { Ag-28Cu-4Ti }+\mathrm{TiN} \\
\text { nanoparticles }\end{array}$ & $800-900{ }^{\circ} \mathrm{C}, 10-60 \mathrm{~min}$ & $\begin{array}{l}\text { Shear strength: } 229 \mathrm{MPa} \text { at } \\
2 \mathrm{vol} \% \mathrm{TiN}, 900{ }^{\circ} \mathrm{C}, 10 \mathrm{~min}\end{array}$ & $\begin{array}{l}\text { Metal-ceramic } \\
\text { brazing }\end{array}$ & {$[82]$} \\
\hline $\begin{array}{c}\mathrm{Al} 4045 \\
(2016)\end{array}$ & Al4045 & $\begin{array}{l}\mathrm{Al}-12 \mathrm{Si}-20 \mathrm{Cu}-(0-0.1) \\
\mathrm{ZrO} 2(\mathrm{wt} \%)\end{array}$ & Induction brazing, $550^{\circ} \mathrm{C}, 30 \mathrm{~min}$ & $\begin{array}{c}\text { Shear strength: 6-MPa, } 0.05 \mathrm{wt} \% \\
\qquad \mathrm{ZrO}_{2}\end{array}$ & $\begin{array}{l}\text { Automotive } \\
\text { vehicles }\end{array}$ & {$[22]$} \\
\hline $\begin{array}{l}\text { Cubic BN } \\
\text { (2016) }\end{array}$ & $\begin{array}{l}\text { AISI } 1045 \\
\text { steel }\end{array}$ & $\begin{array}{c}\left(\mathrm{Ag}_{72} \mathrm{Cu}_{28}\right)_{95} \mathrm{Ti}_{5} / \mathrm{TiB}_{2} \\
(\mathrm{wt} \%)\end{array}$ & $920^{\circ} \mathrm{C}, 5 \mathrm{~min}$ & Shear strength: $50-78 \mathrm{MPa}$ at $8 \mathrm{wt} \%$ & $\begin{array}{c}\text { Cubic BN } \\
\text { superabrasive } \\
\text { wheels }\end{array}$ & [83] \\
\hline $\begin{array}{l}\text { Cubic BN } \\
\text { (2016) }\end{array}$ & $\begin{array}{l}\text { AISI } 1045 \\
\text { steel }\end{array}$ & $\begin{array}{c}\left(\mathrm{Ag}_{72} \mathrm{Cu}_{28}\right)_{95} \mathrm{Ti}_{5} / \mathrm{TiN} \\
(\mathrm{wt} \%)\end{array}$ & $920^{\circ} \mathrm{C}, 5 \mathrm{~min}$ & Shear strength: $50128 \mathrm{MPa}$ at $8 \mathrm{wt} \%$ & $\begin{array}{l}\text { Cubic BN } \\
\text { superabrasive } \\
\text { wheels }\end{array}$ & [83] \\
\hline $\begin{array}{l}\text { Cubic BN } \\
\text { (2016) }\end{array}$ & $\begin{array}{l}\text { AISI } 1045 \\
\text { steel }\end{array}$ & $\begin{array}{c}\left(\mathrm{Ag}_{72} \mathrm{Cu}_{28}\right)_{95} \mathrm{Ti}_{5} / \mathrm{TiC} \\
(\mathrm{wt} \%)\end{array}$ & $920^{\circ} \mathrm{C}, 5 \mathrm{~min}$ & Shear strength: $48 \mathrm{MPa}$ at $8 \mathrm{wt} \%$ & $\begin{array}{l}\text { Cubic BN } \\
\text { superabrasive } \\
\text { wheels }\end{array}$ & [83] \\
\hline $\begin{array}{l}\text { WC-Co } \\
(2017)\end{array}$ & AISI 4145 & Cu-37.9Zn-9.5Ni (wt\%) & $\begin{array}{c}\text { Induction brazing, } 960^{\circ} \mathrm{C}, 1-12.5 \\
\min \end{array}$ & Shear strength: $412 \mathrm{MPa}, 5 \mathrm{~min}$ & Cutting tools & [84] \\
\hline $\begin{array}{c}\text { C-C } \\
\text { composite } \\
(2017)\end{array}$ & $\mathrm{Nb}$ & $\begin{array}{l}\text { Graphene-reinforced } \mathrm{Cu} \\
\text { foam }\end{array}$ & $880^{\circ} \mathrm{C}, 10 \mathrm{~min}$ & Shear strength: $43 \mathrm{MPa}$ & $\begin{array}{l}\text { Thermal } \\
\text { insulation in } \\
\text { aircrafts }\end{array}$ & {$[85]$} \\
\hline $\begin{array}{c}\text { Galvanized } \\
\text { steel } \\
(2017)\end{array}$ & $\begin{array}{l}\text { Galvanized } \\
\text { steel }\end{array}$ & $\mathrm{Cu}-3 \mathrm{Si}(\mathrm{wt} \%)$ & $\begin{array}{l}\text { Laser arc brazing, 50-80 A, 60-90 } \\
\mathrm{cm} / \mathrm{min}\end{array}$ & $\begin{array}{l}\text { Transverse face bend test, strength: } \\
17.9 \mathrm{MPa}, 70 \mathrm{~A}-80 \mathrm{~cm} / \mathrm{min}\end{array}$ & $\begin{array}{l}\text { Automotive } \\
\text { parts }\end{array}$ & [86] \\
\hline $\begin{array}{l}\mathrm{Al}_{2} \mathrm{O}_{3} \\
(2018)\end{array}$ & TiAl & Ag-21Cu-4.5Ti (wt\%) & $\begin{array}{l}\text { Vacuum brazing, } 860-940{ }^{\circ} \mathrm{C}, 1-15 \\
\min \end{array}$ & Strength: $49 \mathrm{MPa}$ at $900^{\circ} \mathrm{C} / 5 \mathrm{~min}$ & $\begin{array}{c}\text { High- } \\
\text { temperature } \\
\text { optical } \\
\text { windows } \\
\end{array}$ & [87] \\
\hline $\begin{array}{l}\text { ZrC-SiC } \\
(2018)\end{array}$ & Ti-6Al-4V & $\mathrm{Ti}-12 \mathrm{Cu}-13 \mathrm{Ni}($ at $\%)$ & $930-990^{\circ} \mathrm{C}$ for $10 \mathrm{~min}$ & $\begin{array}{c}\text { Shear strength }=47 \mathrm{MPa} \text { at } 970^{\circ} \mathrm{C}, \\
10 \mathrm{~min}\end{array}$ & $\begin{array}{l}\text { High- } \\
\text { temperature } \\
\text { shielding and } \\
\text { barriers in } \\
\text { nuclear } \\
\text { reactors }\end{array}$ & {$[88]$} \\
\hline
\end{tabular}


Table 3. Cont.

\begin{tabular}{|c|c|c|c|c|c|c|}
\hline \multicolumn{2}{|c|}{ Contact Materials } & \multirow{2}{*}{$\begin{array}{c}\text { Brazing Filler } \\
\text { Ni-14Cr-10P (wt } \%)\end{array}$} & \multirow{2}{*}{$\begin{array}{c}\text { Brazing Condition } \\
950{ }^{\circ} \mathrm{C}, 5-40 \mathrm{~min}\end{array}$} & \multirow{2}{*}{$\begin{array}{c}\text { Strength (MPa) } \\
\begin{array}{c}\text { Compressive strength }=0.0006 \mathrm{MPa} \\
\text { at } 950{ }^{\circ} \mathrm{C}, 10 \mathrm{~min}\end{array}\end{array}$} & \multirow{2}{*}{$\begin{array}{c}\text { Application } \\
\text { Abrasive tools }\end{array}$} & \multirow{2}{*}{$\begin{array}{l}\text { Ref. } \\
{[32]}\end{array}$} \\
\hline $\begin{array}{l}\text { AISI1045 } \\
(2018)\end{array}$ & AlSI1045 & & & & & \\
\hline $\begin{array}{l}\text { Inconel } \\
718 \\
(2018)\end{array}$ & Inconel 718 & $\begin{array}{l}\mathrm{Ni}-\mathrm{Mn}-\mathrm{Fe}-\mathrm{Co}_{0.25}-\mathrm{Cu}_{1.75} \\
\quad(\mathrm{at} \%), \mathrm{HEA} \text { filler }\end{array}$ & Laser brazing, 350-400 W & Shear strength $220 \mathrm{MPa}, 400 \mathrm{~W}$ & Jet engine parts & [89] \\
\hline $\begin{array}{l}\text { CoCrFeMnNi } \\
\text { HEA } \\
(2019)\end{array}$ & $\begin{array}{l}\text { CoCrFeMnNi } \\
\text { HEA }\end{array}$ & $\begin{array}{l}\mathrm{Ni}-7 \mathrm{Cr}-3.125 \mathrm{~B}-4.5 \mathrm{Si} \\
(\mathrm{wt} \%)\end{array}$ & $\begin{array}{c}\text { Infrared brazing, } 1020-1050{ }^{\circ} \mathrm{C}, 3-10 \\
\min \end{array}$ & $\begin{array}{c}310 \mathrm{MPa}, 1020^{\circ} \mathrm{C}, 10 \mathrm{~min}, 319 \mathrm{MPa}: \\
1050^{\circ} \mathrm{C}, 10 \mathrm{~min}\end{array}$ & $\begin{array}{c}\text { Heat } \\
\text { exchangers }\end{array}$ & [90] \\
\hline $\begin{array}{l}\text { CoCrFeMnNi } \\
\text { HEA } \\
(2019)\end{array}$ & $\begin{array}{l}\text { CoCrFeMnNi } \\
\text { HEA }\end{array}$ & $\begin{array}{c}\text { Ni-16Cr-32Fe-1.5Si-0.5B- } \\
\text { 6P-1.5Mo } \\
(w t \%)\end{array}$ & $\begin{array}{l}\text { Infrared brazing, } 1050-1080{ }^{\circ} \mathrm{C}, 5-10 \\
\min \end{array}$ & $\begin{array}{c}321 \mathrm{MPa}, 1050^{\circ} \mathrm{C}, 10 \mathrm{~min}, 271 \mathrm{MPa} \text {, } \\
1080^{\circ} \mathrm{C}, 10 \mathrm{~min}\end{array}$ & $\begin{array}{c}\text { Heat } \\
\text { exchangers }\end{array}$ & [90] \\
\hline $\begin{array}{l}(2019) \\
\text { 6A01-T5 } \\
\text { Al alloy }\end{array}$ & $\begin{array}{l}\text { Austenitic } \\
\text { stainless } \\
\text { steel }\end{array}$ & $\begin{array}{l}\text { ER-5183 filler wire } \\
\text { (Al-Mg-4.5 Mn, wt\%) }\end{array}$ & $\begin{array}{l}\text { Hybrid laser-cold metal transfer } \\
\text { brazing; laser power, } 1.9-2.2 \mathrm{~kW} \\
\text { welding speed, } 0.8-1.4 \mathrm{~m} / \mathrm{min}\end{array}$ & $\begin{array}{c}\text { Tensile strength: } 188.7 \mathrm{MPa} \text {, at } 1.2 \\
\mathrm{~m} / \mathrm{min}, 2 \mathrm{~kW}\end{array}$ & $\begin{array}{l}\text { Automotive } \\
\text { applications }\end{array}$ & [91] \\
\hline $\begin{array}{c}\mathrm{Ag}- \\
\mathrm{SnO}_{2} / \mathrm{Ag} \\
(2020)\end{array}$ & $\mathrm{Cu} /$ Steel $/ \mathrm{Cu}$ & Cu-15Ag-5P (wt\%) & Induction brazing, $630-780^{\circ} \mathrm{C}$ & $\mathrm{N} / \mathrm{A}$ & Metal forming & [92] \\
\hline $\begin{array}{l}\text { Inconel } \\
718 \\
(2021)\end{array}$ & Inconel 718 & $\begin{array}{c}\mathrm{Ni}-15.6 \mathrm{Cr}-29.5 \mathrm{Fe}-25 \mathrm{Ge} \\
\text { (at } \%)\end{array}$ & Vacuum brazing, $1100^{\circ} \mathrm{C}, 15-60 \mathrm{~min}$ & $\begin{array}{l}\text { Shear strength: } 269 \mathrm{MPa}, 15 \mathrm{~min} ; 296 \\
\text { MPa, } 60 \mathrm{~min}\end{array}$ & $\begin{array}{l}\text { High- } \\
\text { temperature } \\
\text { corrosion } \\
\text { resistance }\end{array}$ & [93] \\
\hline $\begin{array}{l}\text { Rolled EN } \\
\text { 10025-2- } \\
\text { S235JR } \\
\text { steel (cold } \\
\text { rolled and } \\
\text { galva- } \\
\text { nized) }\end{array}$ & $\begin{array}{l}\text { Rolled EN } \\
\text { 10025-2- } \\
\text { S235JR } \\
\text { steel }\end{array}$ & $\begin{array}{c}\text { CuAl8Ni2Fe2Mn2 and } \\
\text { CuAl9Ni5Fe3Mn2, } \\
\text { CuMn12Ni2, CuSi3Mn, } \\
\text { CuAl5Ni2Mn, and } \\
\text { CuAl7 (wt } \%)\end{array}$ & $\begin{array}{l}\text { Arc brazing: } 111,110-118,170-190 \\
\text { A; } 250,380 \mathrm{~mm} / \mathrm{min}\end{array}$ & $\begin{array}{c}\text { Reaction force/heat input: } \\
\text { CuAl5Ni2Mn }(0.000004 \mathrm{MPa} / \mathrm{kJ} \\
\text { single layer and } 0.000006 \mathrm{MPa} / \mathrm{kJ} \\
\text { multi-layer); CuAl7 }(0.000005 \\
\mathrm{MPa} / \mathrm{kJ} \text { and } 0.0000151 \mathrm{MPa} / \mathrm{kJ}) \text {; } \\
\mathrm{CuMn} 12 \mathrm{Ni} 2(0.0000106 \mathrm{MPa} / \mathrm{kJ} \text { and } \\
0.0000496 \mathrm{MPa} / \mathrm{kJ}) ; \mathrm{CuSi} 3 \mathrm{Mn} \\
(0.000009 \mathrm{MPa} / \mathrm{kJ} \text { and } 0.0000304 \\
\mathrm{MPa} / \mathrm{kJ}) ; \mathrm{CuAl} \mathrm{Ni} 5 \mathrm{Fe} 3 \mathrm{Mn} 2 \\
(0.0000104 \mathrm{MPa} / \mathrm{kJ} \text { and } 0.0000289 \\
\mathrm{MPa} / \mathrm{kJ})\end{array}$ & $\begin{array}{l}\text { Automotive } \\
\text { parts }\end{array}$ & [94] \\
\hline
\end{tabular}

We can see from Table 3 that most of the brazing fillers have been applied to join ceramics to metals as well as ceramics to ceramics. The most popular ceramics include $\mathrm{Al}_{2} \mathrm{O}_{3}$, $\mathrm{SiC}, \mathrm{Si}_{3} \mathrm{~N}_{4}, \mathrm{ZrO}_{2}$, and $\mathrm{BN}$ brazed by $\mathrm{Ag}$ - and $\mathrm{Cu}$-based brazing alloys. Ceramic composites such as $\mathrm{C}$ fiber-reinforced $\mathrm{SiC}$, Y-stabilized $\mathrm{ZrO}_{2}, \mathrm{ZrC}-\mathrm{SiC}$, WC-Co, and C-C composite have also been joined using $\mathrm{Cu}$-, Ti-, or Ni-based brazing alloys. Low-temperature brazing was used especially for aluminum-based brazing fillers using $\mathrm{Al}-, \mathrm{Zn}-$, or Ni- based brazing fillers $[22,32,76]$. However, $\mathrm{Zn}$-based fillers had the issue of a low vapor pressure at high temperatures which causes cracks in joints. Later, Jung et al. [22] developed a low-melting Al-based brazing filler (Al-12Si-20Cu-0.05ZrO $\mathrm{Z}_{2}$ ) for joining automotive components by induction brazing. Al components have been joined to cubic boron nitride ceramic wheels for abrasive applications by Miao et al. [83]. Zahid et al. brazed a sandwich structure made of $\mathrm{Ag}-\mathrm{SnO}_{2} / \mathrm{Ag} / \mathrm{Cu} /$ steel using a Cu-15Ag-5P filler [92]. Different types of galvanized steel sheets have been joined together using $\mathrm{Cu}$-based fillers by laser brazing or laser arc hybrid brazing as mentioned by Sharma et al. [86] and Andreazza et al. [94]. In recent years, some remarkable progress has been made in brazing fillers, such as brazing nanopastes [81], nanocomposite fillers [22,82,83], and graphene-reinforced $\mathrm{Cu}$ foam [85]. Although Ag-based alloys are still in demand $[83,87]$ for their brazing activity and strength, novel alloys are now coming up to occupy the pool of brazing alloys. For example, multiprincipal element alloys known as high-entropy alloys have shown their potential in the brazing area. High-entropy brazing fillers have been used to join superalloys and ceramics recently $[89,90]$. We will elaborate the discussion on these high-entropy brazing fillers in Section 7. In the following sections, we will review the various applications of the different brazing alloys in actual joining applications.

\section{Joining Dissimilar Materials}

The recent developments in brazing technology include joining dissimilar materials to combine the properties of both materials in a single device. Various examples of recent activities in the broad discipline of brazing are as follows. 


\subsection{Ceramic Brazing}

Ceramic brazing is complex and requires the use of active metal fillers for joining. In the literature, most ceramics have been directly brazed using Ag-Cu eutectic fillers [18]. Among the various ceramics, sapphire-to-sapphire $\left(\mathrm{Al}_{2} \mathrm{O}_{3} / \mathrm{Al}_{2} \mathrm{O}_{3}\right)$ bonding is most important for aircraft windows, microelectromechanical systems, and scratch-resistant surfaces. However, the joining of sapphires is cumbersome because of their small size. Sapphires have excellent thermodynamic stability and optical transparency, which are required for most lightweight devices and surface engineering components. The brazing of a sapphire/sapphire couple was performed using a novel bismuth-borate glass filler $\left(50 \mathrm{Bi}_{2} \mathrm{O}_{3}-\right.$ $40 \mathrm{~B}_{2} \mathrm{O}_{3}-10 \mathrm{ZnO}$ in molar\%) [95]. Therefore, the use of such glass brazes can expand this ceramic-to-ceramic direct joining in applications such as scratch-resistant components, micromechanical devices, and aircraft windows. The joining of $\mathrm{SiO}_{2 f} / \mathrm{SiO}_{2}$ glass fibers to silica was achieved using an indium-containing filler metal. This filler helps reduce the required brazing temperature, which further alleviates the residual stresses in the joint to improve the service life [96].

\subsection{Joining of Metallic Glasses and Steel}

Since 2000, bulk metallic glasses (BMGs) have shown great potential in the materials research community because of their impressive mechanical performance and excellent corrosion resistance [97]. However, BMGs are often ignored in brazing because their attractive properties deteriorate significantly after they crystallize while joining. During brazing, the interfacial changes in chemical composition transform the supercooled structure to crystalline, which causes further embrittlement issues. Kim et al. used an active metal $\mathrm{Zn}-\mathrm{Ag}$-Al brazing filler and managed to achieve a robust joint composed of a novel $\mathrm{Cu}_{54} \mathrm{Ni}_{6} \mathrm{Zr}_{22} \mathrm{Ti}_{18} \mathrm{BMG}$ and steel without the evolution of any detrimental phases [98]. The crystallized phases were absent across the brazed joint when using an active brazing filler compared with that using a pure $\mathrm{Zn}$ filler.

\subsection{Joining of C (Graphite) and Superalloys}

The brazing of graphite to superalloys is an important process for the nuclear and petrochemical industries. Carbon structures can provide high thermal shock and hightemperature wear resistance to hard counter-contact superalloys [99]. Various Si-doped Au-based brazing fillers have been utilized to join graphite with Ni-based superalloys [99].

\subsection{Joining of Steel to Rocket Nozzles}

In aircraft applications, boron-free fillers have been developed for the brazing of corrosion-resistant heat exchangers and rocket nozzles. The formation of borides in boroncontaining brazing fillers at the interface results in increased brittleness and eventually leads to complete failure during application. Recent developments in boron-free brazing fillers include Ni-Si-Be alloys for joining austenitic-ferritic steels [100].

\subsection{Joining of Plasma/Fusion Reactor Components}

The development of brazing fillers free from radiation-sensitive components ( $\mathrm{Ni}$ and Co) is essential in joining WC parts in nuclear reactors as hard components to divert plasmafacing components and minimize the contamination of plasma. Researchers have addressed these issues using new brazing fillers composed of pure metal powders and organic binders [101]. Thus, we can conclude that research concerning brazing fillers and joiningrelated technologies is widespread across different sectors. We have highlighted a small part, but it is important to emphasize the significance of brazing in the modern industry.

\section{Emerging Areas in Brazing}

To date, the progress in brazing technology has been limited by various challenges, such as low-melting fillers, residual stress, and cost. However, future requirements in brazing will push these issues beyond the conventional metallurgical principles toward 
other research disciplines to develop complex and novel fillers. Modern portable micromechanical devices, miniaturized semiconductors, and electronic circuits have opened a new direction for nanoscale joining to have the ability to withstand elevated temperature operations. The development of nanoparticle-reinforced fillers for joining metal-ceramic parts is a unique challenge. The various areas for development are described in the following.

\subsection{Joining of Nanostructures and Multilayer Coatings}

Joining at the nanoscale is an interesting area for the next generation of miniaturized micromechanical devices composed of nanoparticles, nanowires, and nanotubes [102]. Current advances in this field are limited by brazing issues at the nanoscale, rather than by the fabrication process of these nanostructures. Weak bonding at the nanoscale level between these components leads to premature device failure, which is a major obstacle in the advancement of nanojoining technology [103]. Some notable developments in this area include vacuum brazing of a network of carbon nanotubes using a Ti-doped Ag-Cu active filler [56] and nanojoining of $\mathrm{Ag}$ nanowires using $\mathrm{Au}_{80} \mathrm{Sn}_{20}$ brazing [104].

Nanowires can be directly brazed or fusion bonded, while the direct fusion of nanoparticles is more difficult. Controlling the melting depth is very tough and often results in full melting of the nanoparticle and coalescence, which defeats the purpose of joining them. Nanoscale joining requires partial melting of nanoparticles and control of the melting depth associated with fusion methods. Previous research reports have shown that nanojoining of two different metal nanostructures can be made feasible using lasers of specific wavelengths [105]. The selection of the laser wavelength is carried out in such a way that the absorption edge of either of the two nanoparticles is matched. The heat distribution of one nanoparticle causes melting of the other while keeping the other nanoparticle in the solid phase. Recently, many studies have been conducted on nanomultilayered structures. Multilayer coating systems with thicknesses at the nanolength scale have been used in microwave ceramic circuits. Hu et al. [106] investigated $\mathrm{Ag} / \mathrm{Ge} / \mathrm{AlN}$ and $\mathrm{Ni} / \mathrm{Al}$ nanomultilayers for low-temperature joining of heat-sensitive materials (Figure 5).

$\mathrm{Ag}$ and Ge do not react as they are immiscible with each other. Ge was added into the multilayers to hinder the surface migration of $\mathrm{Ag}$ atoms while AlN acted as a thermal barrier at higher temperatures (Figure 5a). In contrast, the Ni/ $\mathrm{Al}$ multilayer system is highly reactive and has a lower reactive exothermic temperature at the nanolength scale (Figure $5 b$ ). Therefore, it is used as a local heat source in the brazing of heat-sensitive materials. More recently, nanomultilayer coatings of $\mathrm{Ag}$ with ceramic functional layers (Ag-AlN, Cu-AlN, Cu-W, Al-AlN, AgCu-AlN, AlSi-AlN, etc.) have been developed for lowtemperature nanojoining of heat-sensitive components at reduced temperatures [107-113]. Owing to the nanoscale condition, faster diffusion and mass transport were observed in these nanocoatings at temperatures far below the melting point compared to their bulk counterparts. More investigations are necessary for this nanojoining field to understand the basic principles behind the joining of dissimilar materials and ceramic brazing [102].

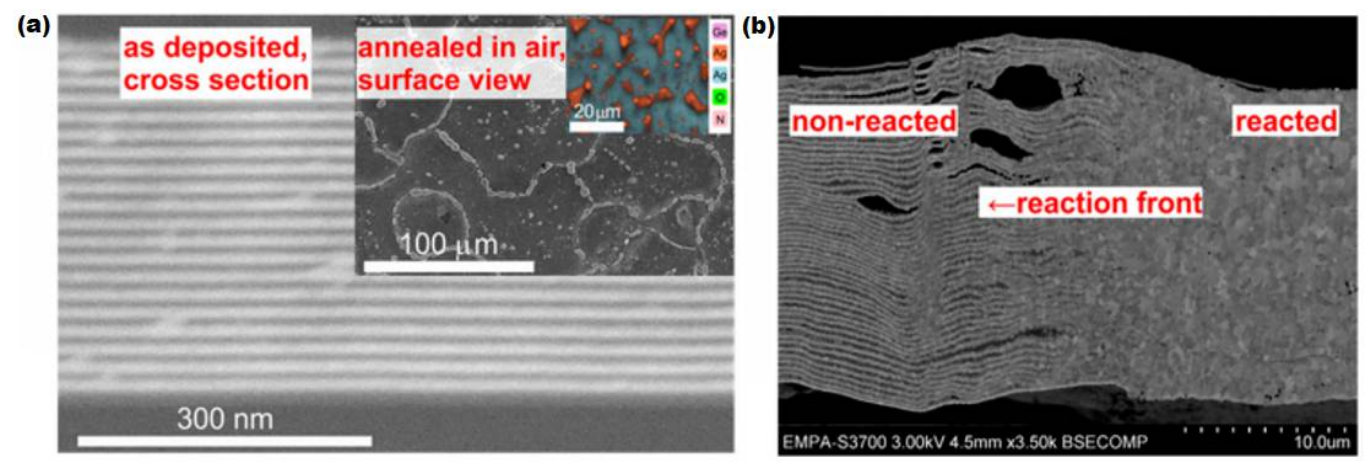

Figure 5. Nanomultilayer filler materials: (a) non-reactive Ag/Ge/AlN nanomultilayer for low-temperature joining and (b) reactive $\mathrm{Ni} / \mathrm{Al}$ nanomultilayer as a local heat source for brazing of heat-sensitive materials [106]. 


\subsection{Joining of Energy Materials}

Another emerging area in the field of brazing research is joining microdevices used as an alternative source of energy such as solid oxide fuel cells (SOFCs). Energy production via SOFCs is a subject of great interest for material engineers because these fuel cells offer excellent improvement in energy efficiency, fewer environmental concerns, and cheaper options compared to other current technologies [114]. In SOFCs, the fuel is oxidized directly to produce electricity where the individual cells (cathode-electrolyte-anode) are stacked (Figure 6).

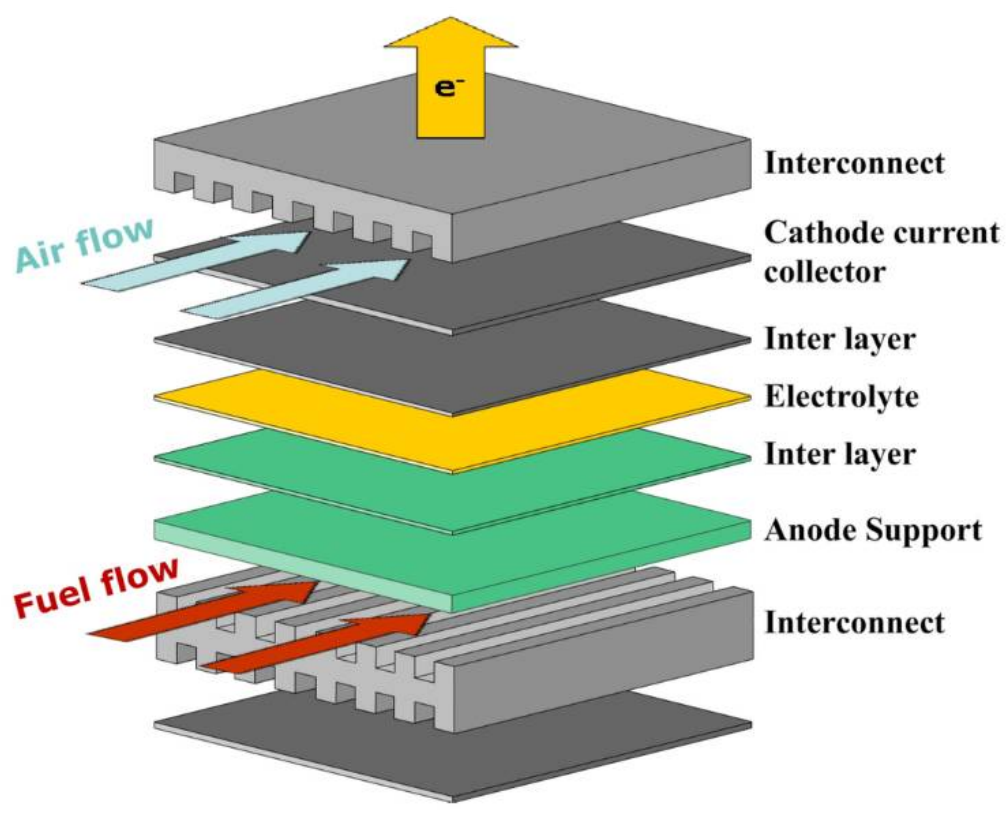

Figure 6. Schematic of SOFC with interconnect-anode-electrolyte-cathode cells. The metal interconnects are brazed to the ceramic electrodes [115].

Each of these stacked small cells in combination produces a large voltage for external work. These cells are brazed using ceramic electrodes to metal interconnects for operating at higher service temperatures $\left(\sim 500-1000^{\circ} \mathrm{C}\right)$. Therefore, active metal fillers are used for SOFC brazing [116]. Other developments include novel brazing methods, such as reactive air brazing (RAB), which was developed by Kim et al. [117]. RAB is preferred over other joining techniques because it is performed in air, and a controlled environment or vacuum is not a big issue [118]. RAB is often carried out above $900{ }^{\circ} \mathrm{C}$ and can sustain the higher operating temperatures required for SOFCs [119]. The active filler for RAB is composed of noble metals such as $\mathrm{Ag}$ and a reactive metal $(\mathrm{Cu})$. The formation of $\mathrm{CuO}$ improves the wetting of Ag over the substrate ceramic. In other studies, porous Ni interlayers have been used as substitutes for $\mathrm{Cu}$ [114]. Joint failure caused by the mismatch of thermal properties between the interlayer and ceramic substrate has led to the development of brazing fillers with modified fractions of the $\mathrm{Al}_{2} \mathrm{TiO}_{5}$ ceramic to control the thermal mismatch [120].

\subsection{Joining of Dissimilar Materials for Lightweight Automotive Applications}

Using lightweight parts in automotive applications is desirable, e.g., replacing steel bodies in cars with $\mathrm{Al}$ alloys can reduce the weight and improve energy efficiency. However, it is a challenging task to join $\mathrm{Al}$ to steel in some areas because of safety concerns. Brazing of $\mathrm{Al}$ to steel is necessary for automotive industries, but this process is difficult [121]. This is mainly because of the poor solid solubility of $\mathrm{Fe}$ in $\mathrm{Al}$, which leads to the generation of FexAly IMCs at the interface joint. These IMCs are harmful and deteriorate the mechanical properties of the joints [122]. Various research activities have been conducted to control these IMCs during $\mathrm{Al}$ brazing. The addition of $\mathrm{Si}$ and $\mathrm{Cu}$ in $\mathrm{Al}$ alloy fillers for the brazing of 5A05 AA to AISI 321 stainless steel [123] was investigated. Mg improves the hot cracking 
sensitivity [122]. Zr was added to a Zn-15Al filler during the brazing of AA 6061 to 304 stainless steel [121].

\subsection{Joining of Dissimilar Materials Using Nanomodified Fillers}

While few studies have yielded considerable improvements in the joint strength and a reduced IMC layer, further research is necessary to produce an acceptable interface at elevated temperatures. To improve the high-temperature joint properties, researchers have used nanoparticles in the fillers to control the IMCs and relax the inherent thermal residual stresses in the joint. Jung et al. developed ceramic-reinforced brazing fillers to join $\mathrm{Al}$ at low temperatures. They reported that the addition of ceramic nanoparticles $\left(\mathrm{ZrO}_{2}\right.$, $\mathrm{La}_{2} \mathrm{O}_{3}$, and $\mathrm{SiC}$ ) not only improved the joint strength and reduced the IMC growth but also decreased the melting point of the brazing filler [69]. This is suitable for saving energy and performing brazing at low temperatures. Other researchers have also used various ceramic reinforcements and metallic nanoparticles to refine the interface thickness. Similar reports have been published on nanocomposite brazing alloys by Miao et al., who recommended the use of TiX additives $\left(\mathrm{TiB}_{2}, \mathrm{TiN}\right.$, and $\left.\mathrm{TiC}\right)$ and graphite in the $\mathrm{Ag}-\mathrm{Cu}-\mathrm{Ti}$ and $\mathrm{Cu}-\mathrm{Sn}$ Ti filler matrix to enhance the brazed joint strength $[83,124]$. The popular nanoceramic additives used in brazing alloys over recent years are $\mathrm{Al}_{2} \mathrm{O}_{3}, \mathrm{SiC}, \mathrm{ZrO}_{2}, \mathrm{CeO}_{2}, \mathrm{La}_{2} \mathrm{O}_{3}$, and $\mathrm{ZrSiO}_{4}$ [125-130]. Despite the advantages of nanocomposite brazing alloys, their use in metal-ceramic brazing is still limited. This can be attributed to the poor dispersion of these additives in the filler melt during solidification, which induces cracks and pores in joints [131-133].

The developments in the area of nanocomposite braze fillers suggest that both the filler composition and control of process parameters such as heat input should also be studied to reduce the IMC fraction and improve the joint strength [134]. Finally, the addition of various alloying elements to the filler metal can sufficiently control the formation of brittle IMCs; however, the specific mechanism of IMC formation is still unclear and requires further investigation.

\subsection{Finite Element Analysis and Simulation}

Bonding between dissimilar materials, especially ceramic joints, has become an important brazing practice in high-temperature applications. Ceramics are known for their excellent mechanical stability at higher temperatures and high resistance to corrosion and wear. However, machining of ceramic joints is difficult due to their brittle nature. Therefore, the thermal stresses build up across the ceramic joints during cooling from brazing to room temperature. Examples in real situations include heat engine components, cutting tools, and thermal barrier coatings. Popular experimental and numerical simulation methods for evaluating thermal residual stresses in ceramic joints include finite element analysis (FEA) $[135,136]$. Experimental tests such as neutron diffraction methods are quite expensive, and narrow brazed joints are difficult to test. In view of these concerns, numerical simulation methods can overcome the shortcomings of experimental tests in analyzing the joint residual stresses. In FEA software, COMSOL Multiphysics is the most widely used software for brazed joint simulation $[137,138]$.

There are various simulation studies that demonstrate that FEA is a potential tool for predicting the location of and measuring the residual stresses in ceramic joints. Zhou et al. [139] investigated a $\mathrm{SiC} / \mathrm{SiC}$ joint brazed with a $\mathrm{Zr}-\mathrm{Cu}$ filler, predicted the residual stresses by FEA, and experimentally verified this by brazing and X-ray diffraction measurements. Their results demonstrated that the residual stress of the joint was axial compressive stress that increased with the increase in the filler thickness and cooling rate. The location of the residual stress across the $\mathrm{SiC} / \mathrm{SiC}$ joint coincided with the FEA simulation. Recently, authors have predicted the compressive stress in a simulated $\mathrm{ZrO}_{2} / \mathrm{Ti}-6 \mathrm{Al}-4 \mathrm{~V}$ joint brazed with an active metal filler (Ag-Cu-Sn-Ti) for dental applications. The authors found a compressive stress of $1477 \mathrm{MPa}$ in the joint which was also confirmed from the FEA investigations. Further, the compressive strains in the joint were found to be located near the 
Ti-6Al-4V alloy, which matched well with the real joint fractured samples [38]. It is believed that current numerical and simulation methods have a great role in the development and assessment of high-temperature ceramic joints.

\section{High-Entropy Fillers}

With the new generation of microdevices and portable automotive vehicles in harsh environments, advanced filler metals have become necessary. Various new metal foams, BMGs, nanocomposite solders, and nanostructures have been used as brazing filler metals $[139,140]$. Despite these developments, researchers in the brazing community have recently begun to divert their attention toward novel alloys called HEAs as filler metals $[13,141]$.

HEAs offer great potential for developing and expanding the brazing sector across all disciplines. Recent investigations have shown promising results in brazing with highentropy fillers [142,143]. Bridges et al. successfully brazed Inconel 718 at $180{ }^{\circ} \mathrm{C}$ using FeCoCuNiMn HEA, with good mechanical properties [142]. Recent works by Tillman et al. demonstrated that doping of $\mathrm{CoCrCuFeNi}$ HEA reduces its melting point to values similar to those of Ni-based fillers [141]. Hardwick et al. designed a novel NiCrFeGeB HEA filler to successfully join Inconel 718 at $1100{ }^{\circ} \mathrm{C}$ for $15 \mathrm{~min}$ [144]. The large number of emerging HEAs can be further investigated for the expansion of HEA fillers, which are still in their early stage of development.

\subsection{High-Entropy Alloys}

HEAs were discovered in 2004 independently by Brian Cantor in the UK and Yeh in Taiwan $[145,146]$. HEAs consist of five or more elements ranging from 5 to 35 at $\%$, with a higher mixing entropy. The first HEA developed was an equiatomic FeCrMn$\mathrm{NiCo}$ face-centered cubic (FCC) solid solution-based alloy, commonly referred to as the Cantor alloy [145]. The phase order diagram of a multicomponent alloy system is depicted in Figure 7, where the locations of the conventional and unexplored HEA systems are highlighted.

\subsection{Thermodynamic Parameters for HEA Effect}

Various rules govern the high-entropy effect and predict the stability of high-entropy phases for almost all compositions between $10^{78}$ and $10^{177}$ distinct alloys [145]. In the literature, an initial guide for predicting solid solution formation is provided in the HumeRothery rules, as follows in Table 4.

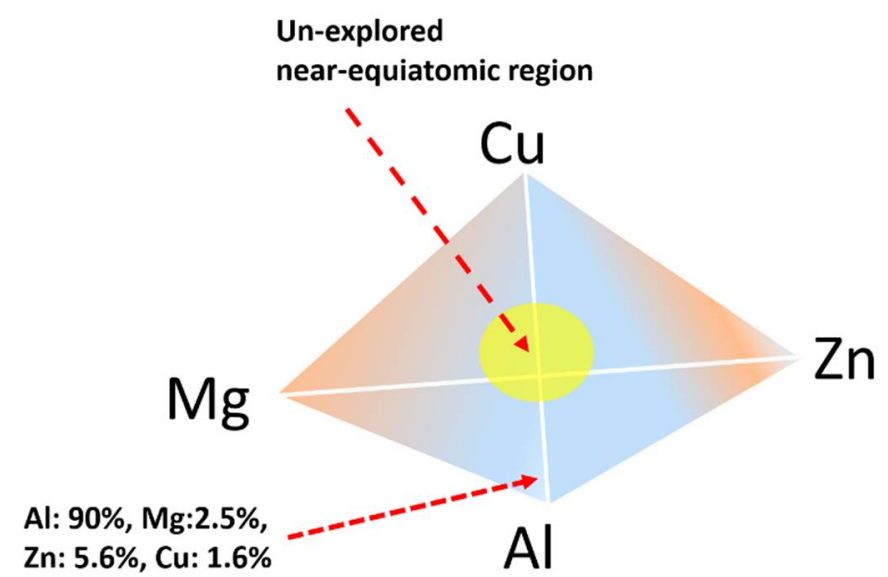

Figure 7. Quaternary phase diagram illustrating conventional (AlMgZnCu alloy) and HEA systems. The central region remains unexplored in most multicomponent element systems. 
Table 4. Thermodynamic parameters for predicting solid solution phases in HEAs [145-147].

\begin{tabular}{|c|c|c|c|}
\hline $\begin{array}{l}\text { Thermodynamic } \\
\text { Parameter }\end{array}$ & Formula & Notations & Phase Stability Condition \\
\hline Atomic size difference & $\begin{array}{c}\delta \\
\sqrt{\sum_{I=1}^{n} c_{i}\left(1-\frac{r_{i}}{r}\right)^{2}} \times 100 \mathrm{r}=\sum_{i=1}^{n} c_{i} r_{i}\end{array}$ & $\begin{array}{c}r_{i}=\text { atomic size of the } i \text { th } \\
\text { element }\end{array}$ & $\delta \leq 6.6 \%$ \\
\hline Enthalpy of mixing & $\Delta \mathrm{H}_{m i x}=4 \sum_{i=1, i \neq j}^{n} \Delta H_{i j}^{m i x} c_{i} c_{j}$ & $\begin{array}{l}c_{i} \text { and } c_{j} \text { are at. } \% \text { of the } i \text { th and } \\
\text { th elements } \\
\Delta H_{i j}^{m i x} \text { is the mixing enthalpy } \\
\text { of equiatomic } i j \text { alloys }\end{array}$ & $-22 \leq \Delta H_{m i x} \leq 7 \mathrm{~kJ} / \mathrm{mol}$ \\
\hline Entropy of mixing & $\Delta \mathrm{S}_{m i x}=-R \sum_{i=1}^{n} c_{i} \ln c_{i}$ & $\begin{array}{c}\mathrm{R}=\text { gas constant }(8.314 \\
\left.\mathrm{J} \cdot \mathrm{mol}^{-1} \cdot \mathrm{K}^{-1}\right)\end{array}$ & $\begin{array}{c}0 \leq \Delta S_{\text {mix }} \leq 8.5,11 \leq \Delta S_{\text {mix }} \leq \\
19.5 \mathrm{~J} /(\mathrm{K} \cdot \mathrm{mol})\end{array}$ \\
\hline $\begin{array}{l}\text { Electronegativity } \\
\text { difference }\end{array}$ & $\begin{array}{c}\Delta \chi=\sqrt{\sum_{I=1}^{n} c_{i}\left(\chi_{i}-\chi\right)^{2}} \\
\chi=\sum_{i=1}^{n} c_{i} \chi_{i}\end{array}$ & $\begin{array}{c}\chi: \text { electronegativity } \\
\chi_{i}: \text { Pauling electronegativity } \\
\text { of the } i \text { th element }\end{array}$ & \\
\hline $\begin{array}{l}\text { Valance electron } \\
\text { concentration }\end{array}$ & $\mathrm{VEC}=\sum_{i=1}^{n} c_{i} V E C_{i}$ & $\begin{array}{c}(V E C) i \text { : number of d-electrons } \\
\text { in the valence shell of the } i \text { th } \\
\text { element }\end{array}$ & $\begin{array}{l}\text { Body-centered cubic }(\mathrm{BCC}) \\
\text { VEC }<6.87 \\
\text { Face-centered cubic }(\mathrm{FCC}): \\
\quad \text { VEC }>8\end{array}$ \\
\hline Interaction parameter & $\begin{aligned} \Omega & =\frac{T_{m} \Delta S_{\operatorname{mix}}}{\left|\Delta H_{m i x}\right|} \\
T_{m} & =\sum_{i=1}^{n} c_{i}\left(T_{m}\right)_{i}\end{aligned}$ & $\begin{array}{l}T_{m}: \text { melting temperature of } \\
\text { the alloy estimated from the } \\
\text { rule of mixtures }\end{array}$ & $\Omega \geq 1.1$ \\
\hline
\end{tabular}

Some researchers have used different parameters to predict the phase formation in HEAs (Figure 8a,b). Guo and Liu [148] used the phase stability criterion related to the valance electron concentration (VEC), while Zhang et al. [147] added two parameters, namely, $\Delta x_{i}$ and VEC. Figure $8 \mathrm{~b}$ illustrates the two different values of $\delta$ and $\Delta H_{\text {mix }}$ parameters used by various researchers to predict the high-entropy effect. Guo et al. concluded that FCC structures are stable at VEC $>8$, while body-centered cubic (BCC) phases are stable when VEC < 6.87 [149]. In 2012, Zhang introduced a new parameter in an attempt to differentiate the solid solution phase and IMC formation criteria known as the $\Omega$ parameter, which involves the melting temperature of the HEA calculated by the rule of mixtures. Zhang argued that when $\Omega>1.1$, the effect of $\Delta S_{\text {mix }}$ is greater than that of $\Delta H_{m i x}$ at $T_{m}$, which further raises the probability of forming a solid solution phase. Based on these criteria, Yang and Zhang proposed $\Omega>1.1$ for $\delta \leq 6.6 \%[150,151]$. The regions of formation of a solid solution, a multiphase, and an amorphous phase are presented in Figure 8.
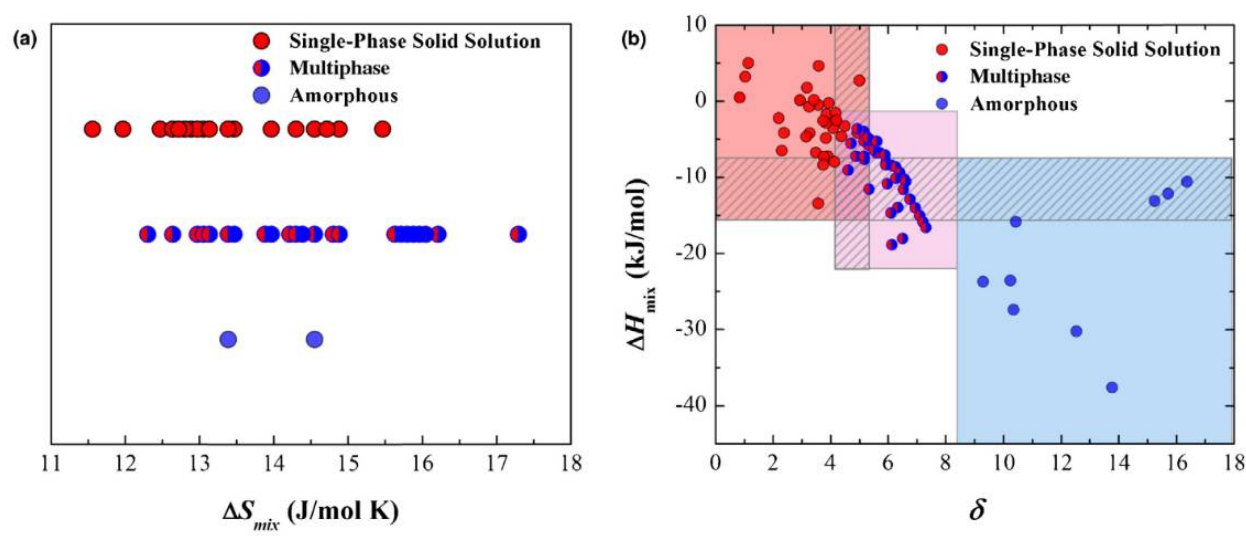

Figure 8. (a) Plot of entropy of mixing of various phase alloy systems. (b) Solid solution phase formation at low atomic size differences and $\delta \leq 6.6 \%, \Omega>1.1[152]$. 
Although none of the compositions and structures meet the standard definition of HEAs as highlighted above, some specific compositions warrant further exploration in brazing.

\subsection{Applications of HEAs in Brazing}

Recent studies on high-entropy fillers include the joining of $\mathrm{ZrB}_{2}-\mathrm{SiC}$ to $\mathrm{GH} 99$ alloy by Zhang et al. using equiatomic CrFeCoNiCu HEA [153]. The superalloy GH99 was combined with a Ti foil to promote wetting without losing the high-entropy effect of the alloy. They inferred that the entropy of mixing of the $\mathrm{Ti} / \mathrm{CrFeCoNiCu}$ filler retains the activity of $\mathrm{Cr}$ and Ti during brazing, and the joint contains a solid solution enriched with $\mathrm{TiC}$ and $\mathrm{B}$ particles, which can enhance the joint strength. Bridges et al. used the same alloy to join Inconel 718 by laser brazing. The authors reported a maximum joint shear strength of $220 \mathrm{MPa}$ at an optimum brazing temperature of $1165^{\circ} \mathrm{C}$ [153].

The doping of $\mathrm{CrFeCoNiCu}$ HEAs was performed by Tillman et al. [154], who added $\mathrm{Ge}, \mathrm{Sn}$, and $\mathrm{Ga}$ to $\mathrm{CrFeCoNiCu}$ and found a decreased melting point and reduced pasty range, which could allow robust joining of Ni superalloys. These authors also used a eutectic $\mathrm{Ni}_{0.73} \mathrm{CrFeCoNi}_{2.1}$ HEA filler to braze Crofer 22 APU interconnecting plates of SOFC to Hf-YSZ ceramics [155]. Similarly, Wang et al. joined SiC to itself using CrFeCoNiCu HEA as a filler. They found that the wetting of the $\mathrm{SiC}$ ceramics was significantly promoted by the activity of $\mathrm{Cr}$, which enhanced the joint strength [156]. Thus, multicomponent HEAs are being vigorously researched for the development of active HEA filler metals.

Several new HEAs have been explored to improve filler metal characteristics and brazing performance. Gao et al. $[157,158]$ highlighted the use of $\mathrm{Fe}_{5} \mathrm{Co}_{20} \mathrm{Ni}_{20} \mathrm{Mn}_{35} \mathrm{Cu}_{20}$ for the joining of Inconel 600 and obtained a high shear strength of $530 \mathrm{MPa}$ when brazed at $1200{ }^{\circ} \mathrm{C} / 90 \mathrm{~min}$ [158]. This is comparable to some of the strength values of conventional $\mathrm{Al}$ alloys. The use of HEAs as fillers has also been tested in the joining of additivemanufactured parts. Sharma et al. recently used an AlZnCuFeSi HEA filler to join an additively manufactured Ti-6Al-4V alloy [159]. The Ti-6Al- $4 \mathrm{~V} / \mathrm{Al}_{2} \mathrm{O}_{3}$ joint microstructure brazed with an AlZnCuFeSi HEA filler is displayed in Figure 9. No defects are observed across the interface. The brazed seam is composed of different regions marked in the reaction layer (I), fusion zone (II), and HEA zone (III), as shown in the figure.

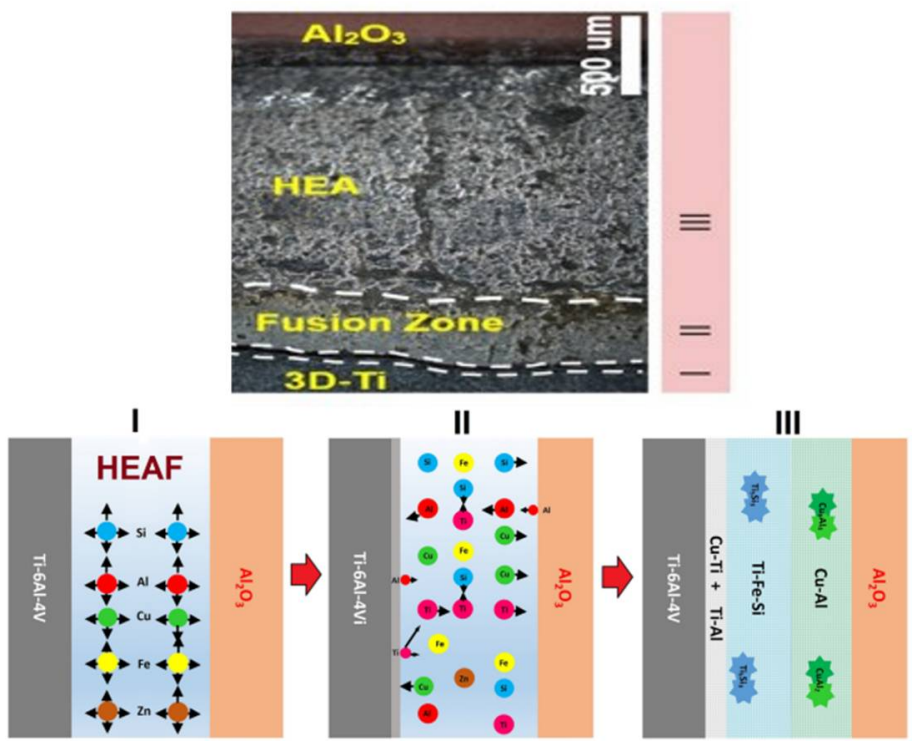

Figure 9. Brazed joint interface of 3D-printed Ti- $6 \mathrm{Al}-4 \mathrm{~V} / \mathrm{Al}_{2} \mathrm{O}_{3}$ system and schematic model of joint formation [159].

The brazed seam was formed in various steps, such as melting of Ti/AlCuSiZnFe, dissolution of $\mathrm{Ti}-\mathrm{Si}$ and $\mathrm{Cu}-\mathrm{Al}$, and solidification of $\mathrm{Ti}-\mathrm{Fe}-\mathrm{Si}, \mathrm{Ti}-\mathrm{Al}$, and $\mathrm{Cu}-\mathrm{Al}$ regions in 
the brazing seam. The authors reported that the use of the $\mathrm{AlZnCuFeSi}$ filler significantly controlled the microstructure of the $\mathrm{Ti} / \mathrm{Al}_{2} \mathrm{O}_{3}$ joint owing to the high-entropy effect [159].

Recently, new HEA filler compositions involve Ag-based HEAs to reduce the cost of fillers containing $\mathrm{Au}$ and $\mathrm{Pt}$ [160] and low-temperature brazing applications [161]. Lowtemperature joining was successfully achieved by Pu et al. using SnBiInZn HEA with a melting temperature of $80^{\circ} \mathrm{C}$ [162]. The filler showed reasonable wetting and strength (19-28 MPa) for microelectronic applications. Hardwick et al. [144] further developed a $\mathrm{NiCrFeGeB}$ filler, which was not equiatomic; however, they modified the composition using the CALPHAD approach to improve the filler properties. Previous studies have highlighted the various merits of HEAs in ceramic brazing [12]. Nevertheless, the use of multicomponent HEAs as fillers is still limited and further research is required in this area.

According to the global market report of Technavio, brazing fillers are the cost-effective choice for the automotive and aerospace markets because of the development of novel alloys and manufacturing technology for vehicles [163]. Brazing fillers provide a better strength and toughness to auto-parts. As a result, brazing fillers are also attractive for aerospace industries in the manufacturing of components operating at high temperature, pressure, and corrosive atmospheres. Examples in aerospace applications include turbines, heat exchangers, and sub-assembly of sensor components. Therefore, rising demands in aerospace industries are expected to boost market growth in the near future. Recycling of Al alloys and development and implementation of economical manufacturing and process automation technologies are expected drive the market growth of brazing fillers. In addition to these, advances in metal-ceramic brazing are other major factors to boost the global brazing market. The global brazing market is expected to rise at a compound annual growth rate (CAGR) of over 5\% during the period 2019-2023 according to the latest market research report by Technavio. The market of global brazing alloys is categorized by application segments into automotive, electrical and electronics, aerospace, and others according to the geographical regions in the Asia-Pacific, Europe, North America, South America, and Middle Eastern countries. The Asia-Pacific region is expected to maintain its dominance in the brazing market due to the rise in brazing applications in the automotive, aerospace, and construction industries. The brazing materials market is primarily split into the following: automobile, aviation, oil and gas, electrical industry, household appliances, power distribution. The end uses of this market cover the following segments: silver brazing alloys, copper brazing alloys, aluminum brazing alloys, and nickel brazing alloys, in the key regions covered in North America (the United States, Canada, and Mexico), Europe (Germany, the UK, France, Italy, Russia, Turkey, etc.), Asia-Pacific (China, Japan, Korea, India, Australia, Indonesia, Thailand, Philippines, Malaysia, and Vietnam), South America (Brazil, Argentina, Columbia, etc.), and the Middle East and Africa (Saudi Arabia, UAE, Egypt, Nigeria, and South Africa).

\section{Summary and Conclusions}

This study presented an overview of the current status and progress in dissimilar material joining and brazing fillers, including the recently discovered HEAs. Brazing is a versatile joining technology that covers an array of diverse industrial applications, particularly in high-temperature microelectronic packaging, energy material design, and biomedical applications. The major advances in this area are focused on the development of novel fillers, nanoparticle-reinforced brazing alloys, BMG fillers, active metal brazing fillers, nanomultilayers, and fusion brazing through laser radiation. The improvement in the brazing process and fillers for advanced joining applications is mostly in active metal brazing, especially for ceramic brazing. The use of nanoparticle additives has shown significant improvements in the wetting and refinement of reaction compounds, but their dispersion in the filler melt is a serious concern. The recently discovered HEAs have demonstrated impressive potential in brazing owing to their sluggish diffusion effects and the formation of solid solution phases rather than reaction compounds. Diffusion bonding is often not understood for HEAs, and the diffusion of constituent elements 
is still unclear, which can further be exploited to expand the scope of low-cost brazing materials for advanced manufacturing. A better understanding of the high-entropy effect and wettability of HEAs is required to effectively apply them as fillers for dissimilar material joining.

Funding: This work was supported by the National Research Foundation of Korea (NRF) grant funded by the Korean government (MSIT) (No. 2021R1A2C1005478).

Institutional Review Board Statement: Not applicable.

Informed Consent Statement: Not applicable.

Data Availability Statement: Not applicable.

Conflicts of Interest: The author declares no conflict of interest.

\section{References}

1. Roberts, P. Industrial Brazing Practice, 2nd ed.; CRC Press: Boca Raton, FL, USA, 2013.

2. American Welding Society. Brazing Handbook, 5th ed.; American Welding Society: Miami, FL, USA, 2007.

3. Lee, S.J.; Sharma, A.; Lee, J.; Jung, D.H.; Jung, J.P. Influence of Arc Brazing Parameters on Microstructure and Joint Properties of Electro-Galvanized Steel. Metals 2019, 9, 1006. [CrossRef]

4. Zhou, W.; Ai, S.; Chen, M.; Zhang, R.; He, R.; Pei, Y.; Fang, D. Preparation and thermodynamic analysis of the porous ZrO2/(ZrO2 + Ni) functionally graded bolted joint. Compos. Part B 2015, 82, 13-22. [CrossRef]

5. Zhou, W.; Zhang, R.; Ai, S.; He, R.; Pei, Y.; Fang, D. Load distribution in threads of porous metal-ceramic functionally graded composite joints subjected to thermomechanical loading. Compos. Struct. 2015, 134, 680-688. [CrossRef]

6. Koleňák, R.; Prach, M. Research of Joining Brittle Nonmetallic Materials with an Active Solder. Adv. Mater. Sci. Eng. 2014, 2014, 729135. [CrossRef]

7. Jia, J.H.; Wang, Z.H.; Yao, D.F.; Tu, S.-T. Brazing Coupling Performance of Piezoelectric Waveguide Transducers for the Monitoring of High Temperature Components. Sensors 2020, 21, 94. [CrossRef]

8. Sharma, A.; Ahn, B. Brazeability, Microstructure, and joint characteristics of ZrO2/Ti-6Al-4V brazed by Ag-Cu-Ti filler rein-forced with cerium oxide nanoparticles. Adv. Mater. Sci. Eng. 2019, 2019, 8602632. [CrossRef]

9. Rybaulin, V.M.; Skorobatyuk, A.V.; Mikitas, A.V. Brazing of absorbers of planar solar heating collectors produced from mate-rials of the $\mathrm{Cu}-\mathrm{CuZn}$ system. Welding Int. 2016, 30, 142-149. [CrossRef]

10. Murty, B.S.; Yeh, J.W.; Ranganathan, S. High. Entropy Alloys, 1st ed.; Butterworth-Heinemann: London, UK, 2014.

11. Sharma, A. High Entropy Alloy Coatings and Technology. Coatings 2021, 11, 372. [CrossRef]

12. Sharma, A.; Duraigina, K.S. (Eds.) High-entropy alloys for micro- and nano-joining applications. In Engineering Steels and High Entropy Alloys; IntechOpen: Rijeka, Croatia, 2020; pp. 1-14.

13. Luo, D.; Xiao, Y.; Hardwick, L.; Snell, R.; Way, M.; Morell, X.S.; Livera, F.; Ludford, N.; Panwisawas, C.; Dong, H.; et al. High Entropy Alloys as Filler Metals for Joining. Entropy 2021, 23, 78. [CrossRef]

14. Way, M.; Willingham, J.; Goodall, R. Brazing filler metals. Int. Mater. Rev. 2020, 65, 257-285. [CrossRef]

15. Arenas, M.; Acoff, V.; Reddy, R. Physical properties of selected brazing filler metals. Sci. Technol. Weld. Join. 2004, 9, 423-429. [CrossRef]

16. Taie, A.K.A.; Ateia, A.A. An experimental study of clearance width effects on the strength of a carbon steel brazed joint. JKAU Eng. Sci. 2007, 18, 57-72. [CrossRef]

17. Eustathopoulos, N.; Voytovych, R. The role of reactivity in wetting by liquid metals: A review. J. Mater. Sci. 2016, 51, $425-437$. [CrossRef]

18. Mishra, S.; Sharma, A.; Jung, D.H.; Jung, J.P. Recent Advances in Active Metal Brazing of Ceramics and Process. Met. Mater. Int. 2020, 26, 1087-1098. [CrossRef]

19. Akselsen, O. Advances in brazing of ceramics. J. Mater. Sci. 1992, 27, 1989-2000. [CrossRef]

20. Shin, J.; Sharma, A.; Jung, D.-H.; Jung, J.P. Effect of Sn Content on Filler and Bonding Characteristics of Active Metal Brazed $\mathrm{Cu} / \mathrm{Al} 2 \mathrm{O} 3$ Joint. Korean J. Met. Mater. 2018, 56, 366-374. [CrossRef]

21. Sharma, A.; Lim, D.-U.; Jung, J.-P. Microstructure and brazeability of $\mathrm{SiC}$ nanoparticles reinforced Al-9Si-20Cu produced by induction melting. Mater. Sci. Technol. 2016, 32, 773-779. [CrossRef]

22. Sharma, A.; Roh, M.-H.; Jung, D.-H.; Jung, J.-P. Effect of $\mathrm{ZrO}^{2}$ Nanoparticles on the Microstructure of Al-Si-Cu Filler for Low-Temperature Al Brazing Applications. Met. Mater. Trans. A 2016, 47, 510-521. [CrossRef]

23. Chen, S.-H.; Li, L.; Chen, Y.-B.; Liu, D.-J. Si diffusion behavior during laser welding-brazing of Al alloy and Ti alloy with Al-12Si filler wire. Trans. Nonferrous Met. Soc. China 2010, 20, 64-70. [CrossRef]

24. Cao, R.; Sun, J.H.; Chen, J.H. Mechanisms of joining aluminium A6061-T6 and titanium Ti-6Al-4V alloys by cold metal transfer technology. Sci. Technol. Weld. Join. 2013, 18, 425-433. [CrossRef]

25. Chen, W. Microstructure and mechanical properties of tungsten inert gas welded-brazed Al/Ti joints. Sci. Technol. Weld. Join. 2016, 21, 547-554. [CrossRef] 
26. Zhu, Z.; Lee, K.Y.; Wang, X. Ultrasonic welding of dissimilar metals, AA6061 and Ti6Al4V. Int. J. Adv. Manuf. Technol. 2012, 59, 569-574. [CrossRef]

27. Winiowski, A.; Majewski, D. Braze Welding TIG of Titanium and Aluminium Alloy Type Al-Mg. Arch. Met. Mater. 2016, 61, 133-142. [CrossRef]

28. Takemoto, T.; Okamoto, I. Intermetallic compounds formed during brazing of titanium with aluminium filler metals. J. Mater. Sci. 1988, 23, 1301-1308. [CrossRef]

29. Zhong, Z.; Hou, G.; Zhu, Z.; Wang, Z.; Wang, G.; Wu, Y. Microstructure and mechanical strength of SiC joints brazed with Cr3C2 particulate reinforced Ag-Cu-Ti brazing alloy. Ceram. Int. 2018, 44, 11862-11868. [CrossRef]

30. Valenza, F.; Gambaro, S.; Muolo, M.L.; Salvo, M.; Casalegno, V. Wetting of SiC by Al-Ti alloys and joining by in-situ formation of interfacial Ti3Si(Al)C 2 . J. Eur. Ceram. Soc. 2018, 38, 3727-3734. [CrossRef]

31. Tian, W.B.; Sun, Z.M.; Zhang, P.; Zhang, Y.M.; Shi, J. Brazing of silicon carbide ceramics with Ni-Si-Ti powder mixtures. J. Aust. Ceram. Soc. 2017, 53, 511-516. [CrossRef]

32. Chen, J.; Mu, D.; Liao, X.; Huang, G.; Huang, H.; Xu, X.; Huang, H. Interfacial microstructure and mechanical properties of synthetic diamond brazed by Ni-Cr-P filler alloy. Int. J. Refract. Met. Hard Mater. 2018, 74, 52-60. [CrossRef]

33. Mukhopadhyay, P.; Simhan, D.R.; Ghosh, A. Challenges in brazing large synthetic diamond grit by Ni-based filler alloy. J. Mater. Process. Technol. 2017, 250, 390-400. [CrossRef]

34. Mukhopadhyay, P.; Ghosh, A. On bond wear, grit-alloy interfacial chemistry and joint strength of synthetic diamond brazed with Ni-Cr-B-Si-Fe and Ti activated Ag-Cu filler alloys. Int. J. Refract. Met. Hard Mater. 2018, 72, 236-243. [CrossRef]

35. Zhang, X.; Liu, G.; Tao, J.; Guo, Y.; Wang, J.; Qiao, G. Brazing of WC-8Co cemented carbide to steel using Cu-Ni-Al alloys as filler metal: Microstructures and joint mechanical behavior. J. Mater. Sci. Technol. 2018, 34, 1180-1188. [CrossRef]

36. Ali, M.; Knowles, K.M.; Mallinson, P.M.; Fernie, J.A. Active metal brazing of $\mathrm{Al}_{2} \mathrm{O}_{3}$ to Kovar ${ }^{\circledR}$ (Fe-29Ni-17Co wt.\%) using Copper $\mathrm{ABA}^{\circledR}(\mathrm{Cu}-3.0 \mathrm{Si}-2.3 \mathrm{Ti}-2.0 \mathrm{Al}$ wt.\%). Philos. Mag. 2017, 98, 182-202. [CrossRef]

37. Emadinia, O.; Guedes, A.; Tavares, C.J.; Simões, S. Joining Alumina to Titanium Alloys Using Ag-Cu Sputter-Coated Ti Brazing Filler. Materials 2020, 13, 4802. [CrossRef] [PubMed]

38. Sharma, A.; Kee, S.H.; Jung, F.; Heo, Y.; Jung, J.P. Compressive Strength Evaluation in Brazed ZrO2/Ti6Al4V Joints Using Finite Element Analysis. J. Mater. Eng. Perform. 2016, 25, 1722-1728. [CrossRef]

39. Pimenta, J.S.; Buschinelli, A.J.A.; Nascimento, R.M.D.; Martinelli, A.E.; Remmel, J. Brazing of zirconia to titanium using Ag-Cu and Au-Ni filler alloys. Soldag. Insp. 2013, 18, 349-357. [CrossRef]

40. Li, J.; Liu, Y.; Tan, Y.; Li, Y.; Zhang, L.; Wu, S.; Jia, P. Effect of tin addition on primary silicon recovery in Si-Al melt during solidification refining of silicon. J. Cryst. Growth 2013, 371, 1-6. [CrossRef]

41. Zor, S.; Zeren, M.; Ozkazanc, H.; Karakulak, E. Effect of titanium addition on corrosion properties of Al-Si eutectic alloys. Prot. Met. Phys. Chem. Surfaces 2012, 48, 568-571. [CrossRef]

42. Aucote, J.; Evans, D.W. Effects of excess silicon addition on ductility of Al-0.95\%Mg2Si alloy. Mat. Sci. Technol. 1978, 12, 57-63.

43. Dahle, A.; Nogita, K.; McDonald, S.; Dinnis, C.; Lu, L. Eutectic modification and microstructure development in Al-Si Alloys Mater. Sci. Eng. A 2005, 413-414, 243-248. [CrossRef]

44. Polmear, I.J. Light Alloys From Traditional Alloys to Nanocrystals, 4th ed.; Elsevier-Butterworth Heinemann: London, UK, 2006.

45. Calliari, I.; Ramous, E.; Brunelli, K.; Favaron, P. Characterization of vacuum brazed joints for super- conducting cavities. Microchim. Acta 2004, 147, 141-146. [CrossRef]

46. Paiva, O.C.; Barbosa, M.A. Production, bonding strength and electrochemical behavior of commercially pure $\mathrm{Ti}^{2} / \mathrm{Al}_{2} \mathrm{O}_{3} \mathrm{brazed}$ joints. J. Mat. Sci. 1997, 32, 653-659. [CrossRef]

47. Timsit, R.S.; Janeway, B.J. A novel brazing technique for aluminum. Weld. J. Res. Suppl. 1994, 73, 119s-128s.

48. Andersson, R.; Holm, T.; Wiberg, S.; Astrom, A. Furnace Atmospheres No. 4, Brazing of Metals, Special ed.; Linde Gas: Munich, Germany, 2011; pp. 1-43.

49. Schwartz, M.M. Brazing, 2nd ed.; The Materials Information Society; ASM International: Novelty, OH, USA, 2001.

50. Kee, S.-H.; Park, S.-Y.; Heo, Y.-K.; Jung, J.-P.; Kim, W.-J. Brazing characteristics of $\mathrm{ZrO}_{2}$ and Ti-6Al-4V brazed joints with increasing temperature. J. Korean Acad. Prosthodont. 2012, 50, 169-175. [CrossRef]

51. Kee, S.H.; Xu, Z.; Jung, J.P.; Kim, W.J. Joining of ceramic and metal using active metal brazing. J. Microelectron. Packag. Soc. 2011, $18,1-7$.

52. Liu, Y.; Hu, J.; Zhang, Y.; Guo, Z.; Yang, Y. Joining of Zirconia and Ti-6Al-4V using A Ti-based amorphous filler. J. Mater. Sci. Technol. 2011, 27, 653-658. [CrossRef]

53. Elrefaey, A.; Tillmann, W. Interface Characteristics and Mechanical Properties of the Vacuum-Brazed Joint of Titanium-Steel Having a Silver-Based Brazing Alloy. Met. Mater. Trans. A 2007, 38, 2956-2962. [CrossRef]

54. Kim, H.-J.; Lee, J.Y.; Paik, K.-W.; Koh, K.-W.; Won, J.; Choe, S.; Lee, J.; Moon, J.-T.; Park, Y.-J. Effects of Cu/Al intermetallic compound (IMC) on copper wire and aluminum pad bondability. IEEE Trans. Compon. Packag. Technol. 2003, 26, 367-374. [CrossRef]

55. Kim, J.H.; Yoo, Y.C. Bonding of alumina to metals with Ag-Cu-Zr brazing alloy. J. Mat. Sci. Lett. 1997, 16, 1212-1215. [CrossRef]

56. He, Y.M.; Zhang, J.; Wang, X.; Sun, Y. Effect of brazing temperature on microstructure and mechanical properties of Si3N4/Si3N4 joints brazed with Ag-Cu-Ti + Mo composite filler. J. Mater. Sci. 2010, 46, 2796-2804. [CrossRef] 
57. Yang, M.; He, P.; Lin, T. Effect of brazing conditions on microstructure and mechanical properties of Al2O3/ Ti-6Al-4V alloy joints reinforced by TiB whiskers. J. Mater. Sci. Technol. 2013, 29, 961-970. [CrossRef]

58. Jiang, W.; Gong, J.; Tu, S. Effect of brazing temperature on tensile strength and microstructure for a stainless steel plate-fin structure. Mater. Des. 2011, 32, 736-742. [CrossRef]

59. Ganjeha, E.; Sarkhoshb, H.; Bajgholic, M.E.; Khorsanda, H.; Ghaffarid, M.H. Increasing Ti-6Al-4V brazed joint strength equal to the base metal by Ti and Zr amorphous filler alloys. Mater. Charact. 2012, 71, 31-40. [CrossRef]

60. Davis, J.R. ASM Specialty Handbook: Copper and Copper Alloys; ASM International: Materials Park, OH, USA, 2001.

61. European Commission. Commission regulation (EU) No 494/Off. J. Eur. Union. 2011, 54, 2-5.

62. Okulov, I.V.; Pauly, S.; Kühn, U.; Gargarella, P.; Marr, T.; Freudenberger, J.; Schultz, L.; Scharnweber, J.; Oertel, C.G.; Skrotzki, W.; et al. Effect of microstructure on the mechanical properties of as-cast Ti-Nb-Al-Cu-Ni alloys for biomedical application. Mater. Sci. Eng. C 2013, 33, 4795-4801. [CrossRef] [PubMed]

63. Burghardt, I.; Lüthen, F.; Prinz, C.; Kreikemeyer, B.; Zietz, C.; Neumann, H.-G.; Rychly, J. A dual function of copper in designing regenerative implants. Biomaterials 2015, 44, 36-44. [CrossRef] [PubMed]

64. Kean, W.F.; Lock, C.J.L.; Buchanan, W.W.; Howard-Lock, H.; Hogan, M.G. Gold Toxicity: Chemical, Structural, Biological and Clinical Experimental Issues; Springer Science and Business Media LLC: Berlin/Heidelberg, Germany, 1992; Volume 3, pp. 321-343.

65. Sharma, S.D.A.K.D.A. Pulse Electrodeposition of Lead-Free Tin-Based Composites for Microelectronic Packaging. In Electrodeposition of Composite Materials; InTech: London, UK, 2016.

66. Das, S.; Das, K.; Sharma, A. Pulse Electroplating of Ultrafine Grained Tin Coating. In Electroplating of Nanostructures; InTech: London, UK, 2015.

67. Suganuma, K. Lead Free Soldering in Electronics: Science. Technology, and Environmental Impact; Marcell Dekker Inc.: New York, NY, USA, 2004.

68. Liu, Y.; Pu, L.; Yang, Y.; He, Q.; Zhou, Z.; Tan, C.; Zhao, X.; Zhang, Q.; Tu, K. A high-entropy alloy as very low melting point solder for advanced electronic packaging. Mater. Today Adv. 2020, 7, 100101. [CrossRef]

69. Yang, C.-H.; Zhou, S.; Lin, S.-K.; Nishikawa, H. Development of Sn-Bi-In-Ga quaternary low-temperature solders. In Proceedings of the 2019 International Conference on Electronics Packaging (ICEP), Niigata, Japan, 17-20 April 2019; pp. 367-369.

70. ISO. ISO/TC Brazing_Filler Metals (ISO 17672:2016), 3rd ed.; British Standards Institution: London, UK, 2016.

71. Sharma, A.; Xu, D.E.; Jung, J.P. Effect of different nanoparticles on microstructure, wetting and joint strength of Al-12Si-20Cu braze filler. Mater. Res. Express 2019, 6, 056526. [CrossRef]

72. Chang, S.Y.; Hung, Y.T.; Chuang, T.H. Joining Alumina to Inconel 600 and UMCo-50 Superalloys Using an Sn10Ag4Ti Active Filler Metal. J. Mater. Eng. Perform. 2003, 12, 123-127. [CrossRef]

73. Kozlova, O.; Braccini, M.; Voytovych, R.; Eustathopoulos, N.; Martinetti, P.; Devismes, M.-F. Brazing copper to alumina using reactive CuAgTi alloys. Acta Mater. 2010, 58, 1252-1260. [CrossRef]

74. Liu, Y.Z.; Zhang, L.X.; Liu, C.B.; Yang, Z.W.; Li, H.W.; Feng, J.C. Brazing C/SiC composites and Nb with TiNiNb active filler metal. Sci. Technol. Weld. Join. 2011, 16, 193-198. [CrossRef]

75. Yang, M.; Lin, T.; He, P. Microstructure evolution of Al2O3/Al2O3 joint brazed with Ag-Cu-Ti + B + TiH2 composite filler. Ceram. Int. 2012, 38, 289-294. [CrossRef]

76. Dai, W.; Xue, S.-B.; Lou, J.-Y.; Lou, Y.-B.; Wang, S.-Q. Torch brazing 3003 aluminum alloy with Zn-Al filler metal. Trans. Nonferrous Met. Soc. China 2012, 22, 30-35. [CrossRef]

77. Laik, A.; Mishra, P.; Bhanumurthy, K.; Kale, G.; Kashyap, B. Microstructural evolution during reactive brazing of alumina to Inconel 600 using Ag-based alloy. Acta Mater. 2013, 61, 126-138. [CrossRef]

78. He, Y.; Zhang, J.; Lv, P.; Liu, C. Characterization of the Si3N4/42CrMo joints vacuum brazed with Pd modified filler alloy for high temperature application. Vacuum 2014, 109, 86-93. [CrossRef]

79. Fan, D.; Huang, J.; Wang, Y.; Chen, S.; Zhao, X. Active brazing of carbon fiber reinforced SiC composite and 304 stainless steel with Ti-Zr-Be. Mater. Sci. Eng. A 2014, 617, 66-72. [CrossRef]

80. Zhang, J.; Wang, T.; Liu, C.; He, Y. Effect of brazing temperature on microstructure and mechanical properties of graphite/copper joints. Mater. Sci. Eng. A 2014, 594, 26-31. [CrossRef]

81. Gorji, A.; Simchi, A.; Kokabi, A. Development of composite silver/nickel nanopastes for low temperature joining of yttriastabilized zirconia to stainless steels. Ceram. Int. 2015, 41, 1815-1822. [CrossRef]

82. Wang, T.; Liu, C.; Leinenbach, C.; Zhang, J. Microstructure and strengthening mechanism of Si3N4/Invar joint brazed with TiNp-doped filler. Mater. Sci. Eng. A 2016, 650, 469-477. [CrossRef]

83. Miao, Q.; Ding, W.; Zhu, Y.; Chen, Z.; Fu, Y. Brazing of CBN grains with Ag-Cu-Ti/TiX composite filler-The effect of TiX particles on microstructure and strength of bonding layer. Mater. Des. 2016, 98, 243-253. [CrossRef]

84. Amirnasiri, A.; Parvin, N.; Shafiei Haghshenas, M. Dissimilar diffusion brazing of WC-Co to AISI 4145 steel using RBCuZn-D interlayer. J. Manuf. Process. 2017, 28, 82-93. [CrossRef]

85. Wang, Z.; Wang, G.; Li, M.; Lin, J.; Ma, Q.; Zhang, A.; Zhong, Z.; Qi, J.; Feng, J. Three-dimensional graphene-reinforced Cu foam interlayer for brazing $\mathrm{C} / \mathrm{C}$ composites and $\mathrm{Nb}$. Carbon 2017, 118, 723-730. [CrossRef]

86. Sharma, A.; Lee, S.-J.; Choi, D.-Y.; Jung, J.P. Effect of brazing current and speed on the bead characteristics, microstructure, and mechanical properties of the arc brazed galvanized steel sheets. J. Mater. Process. Technol. 2017, 249, 212-220. [CrossRef] 
87. Liu, X.; Zhang, L.; Sun, Z.; Feng, J. Microstructure and mechanical properties of transparent alumina and TiAl alloy joints brazed using Ag-Cu-Ti filler metal. Vacuum 2018, 151, 80-89. [CrossRef]

88. Shi, J.M.; Zhang, L.X.; Pan, X.Y.; Tian, X.Y.; Feng, J.C. Microstructure evolution and mechanical property of ZrC-SiC/Ti6Al4V joints brazed using Ti-15Cu-15Ni filler. J. Eur. Ceram. Soc. 2018, 38, 1237-1245. [CrossRef]

89. Bridges, D.; Zhang, S.; Lang, S.; Gao, M.; Yu, Z.; Feng, Z.; Hu, A. Laser brazing of a nickel-based superalloy using a Ni-Mn-Fe-Co$\mathrm{Cu}$ high entropy alloy filler metal. Mater. Lett. 2018, 215, 11-14. [CrossRef]

90. Lin, C.; Shiue, R.-K.; Wu, S.-K.; Huang, H.-L. Infrared Brazing of CoCrFeMnNi Equiatomic High Entropy Alloy Using NickelBased Braze Alloys. Entropy 2019, 21, 283. [CrossRef] [PubMed]

91. Chen, Y.; Yang, Z.; Shi, C.; Xin, Z.; Zeng, Z. Laser-CMT Hybrid Welding-Brazing of Al/Steel Butt Joint: Weld Formation, Intermetallic Compounds, and Mechanical Properties. Materials 2019, 12, 3651. [CrossRef]

92. Zahid, A.; Zahid, U. Influence of process parameters on the formation and quality of joining zones during induction brazing. Eng. Res. Express 2020, 2, 025034. [CrossRef]

93. Liam, H.; Pat, R.; Ed, P.; Goodall, R. Development of a novel Ni-based multi-principal element alloy filler metal, using an Alternative melting point depressant. Metall. Mater. Trans. A 2021, 52, 2534-2548.

94. Andreazza, P.; Gericke, A.; Henkel, K.-M. Investigations on arc brazing for galvanized heavy steel plates in steel and shipbuilding. Weld. World 2021, 65, 1199-1210. [CrossRef]

95. Guo, W.; Wang, T.; Lin, T.; Guo, S.; He, P. Bismuth borate zinc glass braze for bonding sapphire in air. Mater. Charact. 2018, 137, 67-76. [CrossRef]

96. Bo, C.; Zou, W.W.; Li, W.W.; Wu, S.B.; Xiong, H.P. Joining of $\mathrm{SiO}_{2} \mathrm{f} / \mathrm{SiO}_{2}$ composite to Ti-6Al4V using Ag-Cu-In-Ti brazing fillers, the joint strengths, and microstructures. Weld. World. 2017, 61, 833-837. [CrossRef]

97. Inoue, A. Stabilization of metallic supercooled liquid and bulk amorphous alloys. Acta Mater. 2000, 48, 279-306. [CrossRef]

98. Kim, J.; Lee, T. Brazing method to join a novel Cu 54 Ni 6 Zr 22 Ti 18 bulk metallic glass to carbon steel. Sci. Technol. Weld. Join. 2017, 22, 714-718. [CrossRef]

99. Jin, W.; He, Y.; Yang, J.; Zhu, D.; Shen, H.; Wang, L.; Gao, Z. Novel joining of dissimilar materials in the graphite/Hastelloy N alloy system using pure Au doped with Si particles. Mater. Charact. 2017, 131, 388-398. [CrossRef]

100. Ivannikov, A.A.; Kalin, B.A.; Sevryukov, O.N.; Penyaz, M.A.; Fedotov, I.V.; Misnikov, V.E.; Tarasova, M.S. Study of the Ni-Si-Be system as a base to create boron-free brazing filler metals. Sci. Technol. Weld. Join. 2018, 23, 187-197. [CrossRef]

101. De Prado, J.; Sanchez, M.; Utrilla, M.; López, M.; Ureña, A. Study of a novel brazing process for W-W joints in fusion applications. Mater. Des. 2016, 112, 117-123. [CrossRef]

102. Zhou, Y.; Hu, A. From Microjoining to Nanojoining. Open Surf. Sci. J. 2010, 3, 32-41. [CrossRef]

103. Cui, Q.; Gao, F.; Mukherjee, S.; Gu, Z. Joining and Interconnect Formation of Nanowires and Carbon Nanotubes for Nanoelectronics and Nanosystems. Small 2009, 5, 1246-1257. [CrossRef]

104. Li, Q.; Chen, Z.; Zhang, X.; Peng, Y.; Ghosh, P.; Yao, G.; Luo, H.; Lv, J.; Qiu, M. Au80Sn20-based targeted noncontact nanosoldering with low power consumption. Opt. Lett. 2018, 43, 4989-4992. [CrossRef]

105. Hu, A.; Janczak-Rusch, J.; Sano, T.; Quintino, L.; Zhou, Y. Nano brazing of Pt-Ag nanoparticles under femtosecond laser irradiation. Nano-Micro Lett. 2013, 5, 88-92.

106. Hu, A.; Janczak-Rusch, J.; Sano, T. Joining Technology Innovations at the Macro, Micro, and Nano Levels. Appl. Sci. 2019, 9, 3568. [CrossRef]

107. Chiodi, M.; Cancellieri, C.; Moszner, F.; Andrzejczuk, M.; Janczak-Rusch, J.; Jeurgens, L.P.H. Massive Ag migration through metal/ceramic nano-multilayers: An interplay between temperature, stress-relaxation and oxygen-enhanced mass transport. J. Mater. Chem. C 2016, 4, 4927-4938. [CrossRef]

108. Lehmert, B.; Janczak-Rusch, J.; Pigozzi, G.; Zuraw, P.; La Mattina, F.; Wojarski, L.; Tillmann, W.; Jeurgens, L.P.H. Copper-Based Nanostructured Coatings for Low-Temperature Brazing Applications. Mater. Trans. 2015, 56, 1015-1018. [CrossRef]

109. Kaptay, G.; Janczak-Rusch, J.; Jeurgens, L.P. Melting Point Depression and Fast Diffusion in Nanostructured Brazing Fillers Confined Between Barrier Nanolayers. J. Mater. Eng. Perform. 2016, 25, 3275-3284. [CrossRef]

110. Sharma, A.; Lee, S.J.; Oh, J.H.; Jung, J.P. AISI 304 Steel Brazing Using A Flexible Brazing Foil Fabricated by Tape Casting Method. Korean J. Met. Mater. 2017, 55, 836-844. [CrossRef]

111. Janczak-Rusch, J.; Chiodi, M.; Cancellieri, C.; Moszner, F.; Hauert, R.; Pigozzi, G.; Jeurgens, L.P.H. Structural evolution of Ag-Cu nano-alloys confined between AlN nano-layers upon fast heating. Phys. Chem. Chem. Phys. 2015, 17, 28228-28238. [CrossRef]

112. Wejrzanowski, T.; Lipecka, J.; Janczak-Rusch, J.; Lewandowska, M. Melting behavior of Al-Si-based nanomultilayers: Experiments and simulations. Appl. Surf. Sci. 2019, 493, 261-270. [CrossRef]

113. Cancellieri, C.; Klyatskina, E.; Chiodi, M.; Janczak-Rusch, J.; Jeurgens, L.P.H. The Effect of Interfacial Ge and RF-Bias on the Microstructure and Stress Evolution upon Annealing of Ag/AlN Multilayers. Appl. Sci. 2018, 8, 2403. [CrossRef]

114. Zhou, Q.; Bieler, T.R.; Nicholas, J.D. Transient porous nickel interlayers for improved silver-based Solid Oxide Fuel Cell brazes. Acta Mater. 2018, 148, 156-162. [CrossRef]

115. Chen, D.; He, H.; Zhang, D.; Wang, H.; Ni, M. Percolation Theory in Solid Oxide Fuel Cell Composite Electrodes with a Mixed Electronic and Ionic Conductor. Energies 2013, 6, 1632-1656. [CrossRef] 
116. Chao, C.L.; Chu, C.L.; Fuh, Y.K.; Hsu, R.Q.; Lee, S.; Cheng, Y.N. Interfacial characterization of nickel-yttriastabilized zirconia cermet anode/interconnect joints with Ag-Pd-Ga active filler for use in solid-oxide fuel cells. Int. J. Hydrogen Energy 2015, 40, 1523-1533. [CrossRef]

117. Kim, J.Y.; Hardy, J.S.; Weil, K.S. Novel Metal-Ceramic Joining for Planar SOFCs. J. Electrochem. Soc. 2005, 152, J52-J58. [CrossRef]

118. Phongpreecha, T.; Nicholas, J.D.; Bieler, T.R.; Qi, Y. Computational design of metal oxides to enhance the wetting and adhesion of silver-based brazes on yttriastabilized-zirconia. Acta Mater. 2018, 152, 229-238. [CrossRef]

119. Chen, H.; Li, L.; Kemps, R.; Michielsen, B.; Jacobs, M.; Snijkers, F.; Middelkoop, V. Reactive air brazing for sealing mixed ionic electronic conducting hollow fibre membranes. Acta Mater. 2015, 88, 74-82. [CrossRef]

120. Kiebach, R.; Engelbrecht, K.; Grahl-Madsen, L.; Sieborg, B.; Chen, M.; Hjelm, J.; Norrman, K.; Chatzichristodoulou, C.; Hendriksen, P.V. An Ag based brazing system with a tunable thermal expansion for the use as sealant for solid oxide cells. J. Power Sources 2016, 315, 339-350. [CrossRef]

121. Yang, J.; Xue, S.; Sekulic, D.P. An Impact of Zirconium Doping of Zn-Al Braze on the Aluminum-Stainless Steel Joints Integrity During Aging. J. Mater. Eng. Perform. 2017, 26, 358-365. [CrossRef]

122. Su, Y.; Hua, X.; Wu, Y. Influence of alloy elements on microstructure and mechanical property of aluminum-steel lap joint made by gas metal arc welding. J. Mater. Process. Technol. 2014, 214, 750-755. [CrossRef]

123. Song, J.; Lin, S.; Yang, C.; Fan, C. Effects of Si additions on intermetallic compound layer of aluminum-steel TIG welding-brazing joint. J. Alloys Compd. 2009, 488, 217-222. [CrossRef]

124. Miao, Q.; Ding, W.; Fu, D.; Chen, Z.; Fu, Y. Influence of graphite addition on bonding properties of abrasive layer of metal-bonded CBN wheel. Int. J. Adv. Manuf. Technol. 2017, 93, 2675-2684. [CrossRef]

125. Sharma, A.; Yu, H.; Cho, I.S.; Seo, H.; Ahn, B. $\mathrm{ZrO}_{2}$ Nanoparticle Embedded Low Silver Lead Free Solder Alloy for Modern Electronic Devices. Electron. Mater. Lett. 2018, 15, 27-35. [CrossRef]

126. Halbig, M.C.; Coddington, B.; Asthana, R.; Singh, M. Characterization of silicon carbide joints fabricated using SiC particulatereinforced Ag-Cu-Ti alloys. Ceram. Int. 2013, 39, 4151-4162. [CrossRef]

127. Yang, J.; Fang, H.Y.; Wan, X. Effects of $\mathrm{Al}_{2} \mathrm{O}_{3}$ particulate contained composite filler materials on the strength of alumina joints. $J$. Mater. Sci. Technol. 2002, 18, 289-290.

128. Sharma, A.; Srivastava, A.K.; Lee, K.; Ahn, B. Impact of Non-Reactive Ceria Nanoparticles on the Wettability and Reaction Kinetics Between Lead-Free Sn-58Bi and Cu Pad. Met. Mater. Int. 2019, 25, 1027-1038. [CrossRef]

129. Sharma, A.; Srivastava, A.K.; Ahn, B. Microstructure, mechanical properties, and drop reliability of $\mathrm{CeO}_{2}$ reinforced Sn-9Zn composite for low temperature soldering. Mater. Res. Express 2019, 6, 056520. [CrossRef]

130. Bhattacharya, S.; Sharma, A.; Das, S.; Das, K. Synthesis and Properties of Pulse Electrodeposited Lead-Free Tin-Based Sn/ZrSiO 4 Nanocomposite Coatings. Met. Mater. Trans. A 2016, 47, 1292-1312. [CrossRef]

131. Moszner, F.; Cancellieri, C.; Becker, C.; Chiodi, M.; Janczak-Rusch, J.; Jeurgens, L.P.H. Nano-Structured Cu/W Brazing Fillers for Advanced Joining Applications. J. Mater. Sci. Eng. B 2016, 6, 226-230. [CrossRef]

132. Bian, H.; Zhang, Q.; Song, Y.; Liu, D.; Liu, J.; Song, X. Microstructure and Mechanical Properties of a SiO2 Ceramic and TC4 Alloy Joint Brazed with a Nanocomposite Filler. J. Mater. Eng. Perform. 2019, 28, 4427-4433. [CrossRef]

133. Busbaher, D.; Liu, W.; Sekulic, D.P. Mechanical properties of nanoparticles reinforced Mo-Ni braze for a dispenser cathode application. In Proceedings of the IVESC 2012, Monterey, CA, USA, 24-26 April 2012; pp. 485-486.

134. Singh, J.; Arora, K.S.; Shukla, D.K. Dissimilar MIG-CMT weld-brazing of aluminium to steel: A review. J. Alloys Compd. 2019, 783, 753-764. [CrossRef]

135. Sun, R.; Zhu, Y.; Guo, W.; Peng, P.; Li, L.; Zhang, Y.; Fu, J.; Li, F.; Zhang, L. Microstructural evolution and thermal stress relaxation of Al2O3/1Cr18Ni9Ti brazed joints with nickel foam. Vacuum 2018, 148, 18-26. [CrossRef]

136. Acevedo, C.; Drezet, J.-M.; Lefebvre, J.P.; D'Alvise, L.; Nussbaumer, A. Residual Stresses in As-Welded Joints: Finite Element Modeling and Neutron Difraction Stress Measurements. Key Eng. Mater. 2011, 488-489, 335-338. [CrossRef]

137. Mackerle, J. Review of pre- and postprocessor programs included in the major commercial general purpose finite element packages. Adv. Eng. Softw. 1983, 5, 43-53. [CrossRef]

138. Anzalone, R.; D'Arrigo, G.; Camarda, M.; Locke, C.; Saddow, S.E.; La Via, F. Advanced Residual Stress Analysis and FEM Simulation on Heteroepitaxial 3C-SiC for MEMS Application. J. Microelectromech. Syst. 2011, 20, 745-752. [CrossRef]

139. Zhou, B.; Zeng, Z.; Cai, Y.; Feng, K. FEM Simulation and Verification of Brazing SiC Ceramic with Novel Zr-Cu Filler Metal. Materials 2019, 12, 3380. [CrossRef] [PubMed]

140. Zhang, J.; Gu, J.; Li, L.; Huan, Y.; Wei, B. Bonding of alumina and metal using bulk metallic glass forming alloy. Int. J. Mod. Phys. B 2009, 23, 1306-1312. [CrossRef]

141. Tillmann, W.; Wojarski, L.; Manka, M.; Timmer, C. Eutectic high entropy alloys-A novel class of materials for brazing applications. In Proceedings of the International Brazing \& Soldering Conference, New Orleans, LA, USA, 15-18 April 2018; pp. 142-148.

142. Gao, M. Development of New High Entropy Alloys for Brazing of Ni-Base Superalloys. Ph.D. Thesis, Colorado School of Mines, Golden, CO, USA, 2017.

143. Filho, F.C.G.; Monteiro, S.N. Welding Joints in High Entropy Alloys: A Short-Review on Recent Trends. Materials 2020, $13,1411$. [CrossRef] 
144. Hardwick, L.; Rodgers, P.; Pickering, E.J. Development of novel nickel-based brazing alloys, utilising alternative melting point depressants and high entropy alloy concepts. In Proceedings of the 12th International Conference on Brazing, High Temperature Brazing and Diffusion Bonding, Aachen, Germany, 21-23 May 2019; pp. 7-17.

145. Cantor, B.; Chang, I.; Knight, P.; Vincent, A. Microstructural development in equiatomic multicomponent alloys. Mater. Sci. Eng. A 2004, 375-377, 213-218. [CrossRef]

146. Yeh, J.-W.; Chen, S.K.; Lin, S.-J.; Gan, J.-Y.; Chin, T.-S.; Shun, T.-T.; Tsau, C.-H.; Chang, S.-Y. Nanostructured High-Entropy Alloys with Multiple Principal Elements: Novel Alloy Design Concepts and Outcomes. Adv. Eng. Mater. 2004, 6, 299-303. [CrossRef]

147. Zhang, Y.; Zuo, T.T.; Tang, Z.; Gao, M.C.; Dahmen, K.A.; Liaw, P.K.; Lu, Z.P. Microstructures and properties of high-entropy alloys. Prog. Mater. Sci. 2014, 61, 1-93. [CrossRef]

148. Guo, S.; Liu, C.T. Phase stability in high entropy alloys: Formation of solid-solution phase or amorphous phase. Prog. Nat. Sci. Mater. Int. 2011, 21, 433-446. [CrossRef]

149. Guo, S.; Ng, C.; Lu, J.; Liu, C.T. Effect of valence electron concentration on stability of fcc or bcc phase in high entropy alloys. J. Appl. Phys. 2011, 109, 103505. [CrossRef]

150. Yang, X.; Zhang, Y. Prediction of high-entropy stabilized solid-solution in multi-component alloys. Mater. Chem. Phys. 2012, 132, 233-238. [CrossRef]

151. Zhang, Y.; Zhou, Y.J.; Lin, J.P.; Chen, G.L.; Liaw, P.K. Solid-Solution Phase Formation Rules for Multi-component Alloys. Adv. Eng. Mater. 2008, 10, 534-538. [CrossRef]

152. Ye, Y.; Wang, Q.; Lu, J.; Liu, C.; Yang, Y. High-entropy alloy: Challenges and prospects. Mater. Today 2016, 19, 349-362. [CrossRef]

153. Zhang, L.; Shi, J.; Li, H.; Tian, X.; Feng, J. Interfacial microstructure and mechanical properties of ZrB2SiC C ceramic and GH99 superalloy joints brazed with a Ti-modified FeCoNiCrCu high-entropy alloy. Mater. Des. 2016, 97, 230-238. [CrossRef]

154. Tillmann, W.; Ulitzka, T.; Wojarski, L.; Manka, M.; Wagstyl, D. Development of high entropy alloys for brazing applications. Weld. World 2020, 64, 201-208. [CrossRef]

155. Tillmann, W.; Wojarski, L.; Stangier, D.; Manka, M.; Timmer, C. Application of the eutectic high entropy alloy Nb0.73CoCrFeNi2.1 for high temperature joints. Weld. World 2020, 64, 1597-1604. [CrossRef]

156. Wang, G.; Yang, Y.; He, R.; Tan, C.; Huttula, M.; Cao, W. A novel high entropy CoFeCrNiCu alloy filler to braze SiC ceramics. J. Eur. Ceram. Soc. 2020, 40, 3391-3398. [CrossRef]

157. Gao, M.C. Progress in High Entropy Alloys. JOM 2014, 67, 2251-2253. [CrossRef]

158. Gao, M.; Schneiderman, B.; Gilbert, S.M.; Yu, Z. Microstructural Evolution and Mechanical Properties of Nickel-Base Superalloy Brazed Joints Using a MPCA Filler. Met. Mater. Trans. A 2019, 50, 5117-5127. [CrossRef]

159. Sharma, A.; Ahn, B. Vacuum brazing of $\mathrm{Al}_{2} \mathrm{O}_{3}$ and $3 \mathrm{D}$ printed Ti6Al4V lap-joints using high entropy driven $\mathrm{AlZnCuFeSi}$ filler. Sci. Rep. 2021, 11, 9345. [CrossRef] [PubMed]

160. Snell, R. The Development of Novel Silver Brazing Alloys. Ph.D. Thesis, University of Sheffield, Sheffield, UK, 2018.

161. Way, M.; Luo, D.; Tuley, R.; Goodall, R. A new high entropy alloy brazing filler metal design for joining skutterudite thermoelectrics to copper. J. Alloys Compd. 2021, 858, 157750. [CrossRef]

162. Pu, L.; He, Q.; Yang, Y.; Zhao, X.; Hou, Z.; Tu, K.N.; Liu, Y. The Microstructure and Mechanical Property of the High Entropy Alloy as a low Temperature Solder. In Proceedings of the 2019 IEEE 69th Electronic Components and Technology Conference (ECTC), Las Vegas, NV, USA, 28-31 May 2019; pp. 1716-1721.

163. Technavio. Global Braze Alloys Market 2019-2023. Growing Recyclability of Braze Alloys to Boost Growth. Available online: www.technavio.com (accessed on 17 June 2021). 